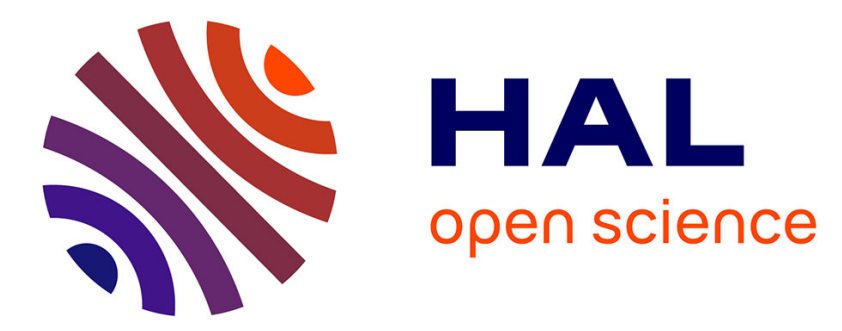

\title{
Photovoltaics with Ferroelectrics: Current Status and Beyond
}

Charles Paillard, Xiaofei Bai, I.C. Infante, Mael Guennou, Grégory Geneste, Alexe M., J. Kreisel, B Dkhil

\section{- To cite this version:}

Charles Paillard, Xiaofei Bai, I.C. Infante, Mael Guennou, Grégory Geneste, et al.. Photovoltaics with Ferroelectrics: Current Status and Beyond. Advanced Materials, 2016, 28, pp.5153. 10.1002/adma;201502515 . hal-01385066

\section{HAL Id: hal-01385066 https://hal.science/hal-01385066}

Submitted on 23 Sep 2020

HAL is a multi-disciplinary open access archive for the deposit and dissemination of scientific research documents, whether they are published or not. The documents may come from teaching and research institutions in France or abroad, or from public or private research centers.
L'archive ouverte pluridisciplinaire HAL, est destinée au dépôt et à la diffusion de documents scientifiques de niveau recherche, publiés ou non, émanant des établissements d'enseignement et de recherche français ou étrangers, des laboratoires publics ou privés. 


\section{Photovoltaic with \\ Ferroelectrics: Current Status and Beyond}

Charles Paillard ${ }^{1}$, Xiaofei Bai ${ }^{1}$, Ingrid Canero Infante ${ }^{1,2}$, Maël

Guennou $^{2}$, Grégory Geneste ${ }^{3}$, Marin Alexe ${ }^{4}$, Jens Kreisel2,5, and

Brahim Dkhil ${ }^{* 1}$

${ }^{1}$ Laboratoire Structures, Propriétés et Modélisation des Solides, CentraleSupélec, CNRS-UMR 8580, Université Paris-Saclay, 92295 Châtenay-Malabry Cedex, France

${ }^{2}$ Materials Research and Technology Department, Luxembourg Institute of Science and Technology, 41 rue du Brill, L-4422 Belvaux, Luxembourg ${ }^{3}$ CEA, DAM, DIF, F-91297 Arpajon, France

${ }^{4}$ Department of Physics, University of Warwick, Coventry CV 4 7AL, United Kingdom ${ }^{5}$ Physics and Materials Science Research Unit, University of Luxembourg, 41 Rue du Brill, L-4422 Belvaux, Luxembourg February 1, 2016

Keywords : Multiferroics, Photovoltaics, Photostriction, Photocatalysis

Abstract
Ferroelectrics carry a switchable spontaneous electric polarization. This
polarization is usually coupled to strain making ferroelectrics good piezoelectrics.
When coupled to magnetism, it leads to the so-called mutliferroic system, a field


being widely investigated since 2003. While ferroelectrics are birefringent and nonlinear optically transparent materials, the coupling of polarization with optical properties has received since 2009 a renewed attention, triggered notably by lowbandgaps ferroelectrics suitable for sunlight spectrum absorption and original photovoltaic effects. Consequently, power conversion efficiencies up to $8.1 \%$ were recently achieved and values of $19.5 \%$ were predicted making photoferroelectrics promising photovoltaic alternatives. This article aims at providing an upto-date review on this emerging and fast progressing field by highlighting several important issues and parameters such as the role of domain walls, ways to tune the band gap, consequences arising from the polarization switchability, the role of defects and contact electrodes as well as the effect of downscaling. Beyond photovoltaicity, other polarization-related processes are also described like light-induced deformation or chemical reaction i.e. photostriction or photocatalysis, respectively. We hope that this initial overview will encourage further avenues to be explored and challenged and, as a byproduct, will inspire other materials research communities, e.g. so-called hybrid halide perovskites.

\section{Introduction}

Ferroelectrics are typically materials with high dielectric constant values, leading to applications as capacitors. They carry a spontaneous electric polarization that can be switched between two or more states using an electric field [1]. This switchability is exploited in ferroelectric-based non-volatile memories and logic devices. Most of the time, the electrical polarization couples strongly with elastic strain, making ferroelectrics also good piezoelectrics suitable as sensors (as in biomaterial detection applications [2]), transducers (as in PZT-based Doppler imaging techniques), Micro-Electro-Mechanical Systems (MEMS) or very precise actuators [3]. More recently, the coupling of polarization and magnetism has led to the field of multiferroics materials, which has attracted a significant interest since 2003 with several fundamental, innovative and applicative issues revealed through reviews $[4,5]$. In the quest for multifunctional materials, the interaction 
with other degrees of freedom, such as light, is now under intense scrutiny. As optical elements, ferroelectrics were essentially considered through their refractive index (or dielectric susceptibility) as optically transparent and birefringent materials with non-linear optical properties of interest for second harmonic generation, phase mixing or electro- and acousto-optical couplings. Since 2009, the coupling of polarization with optical properties in photoferroelectrics has received a renewed attention triggered notably by low bandgaps ferroelectric materials and original photovoltaic effects [6]. Indeed, pioneering work in the early 1970's evidenced an unconventional photovoltaic effect in ferroelectrics due to their non-centrosymmetric nature [7], the so-called Bulk Photovoltaic Effect (BPVE). While the field remained at a standstill for decades, it has received a renewed interest since 2009. Since then, the field has been thriving (see Figure 1), achieving quickly an unprecedented $8.1 \%$ power conversion efficiency (PCE) [8]. While most commercial solar cells are based on the p-n junction principle, they are limited by the so-called Shockley-Queisser limit, which prevent any single p-n junction solar cell from converting more than $33.7 \%$ of the incident light [9]. Such limitations have not been predicted to apply for the BPVE, although the ultimate efficiency for single bandgap absorbers, as predicted by Shockley et al. in the same article [9] should still apply. Work on the theoretical limitations of the BPVE has claimed that the PCE of a single BPVEmaterial based solar cell should be limited by the ratio of the lifetime of nonthermalized non-equilibrium carriers with the lifetime of thermalized nonequilibrium carriers in the conduction, setting the upper PCE limit to $0.01 \%$ [10]. However, such limit has been broken in $\mathrm{BaTiO}_{3}$ thin films, for which a $0.6 \%$ PCE has been recorded [11]. In addition, other effects than the BPVE can come into play when designing ferroelectric-based photovoltaic. For instance, using a semiconductor-based model with a charge distribution in the ferroelectric that includes polarization surface charges, Schottky-induced space charges, and screening charges in the electrodes, it has been estimated that an extremely high 
photovoltaic efficiency up to $19.5 \%$ can be reached in thin films about $1.2 \mathrm{~nm}$ thick sandwiched by electrodes with infinite dielectric constant [12]. Nonetheless, a theoretical upper PCE limit in photoferroelectrics is, to the best of our knowledge, still an open question. Note however that the maximum efficiency any single-bandgap absorber can achieve, known as the ultimate efficiency, has been theoretically set to $44 \%$ by thermodynamic considerations [9]. This estimate relies on the assumptions that the solar cell is at the absolute zero temperature to prevent black body radiation, has a $100 \%$ internal quantum efficiency, that all excited electrons have the same energy (that of the bandgap) and that the full energy of excited electrons can be collected. Under these assumptions, the ideal cell would have a bandgap of $1.1 \mathrm{eV}$. It is worth mentioning that in addition to the PCE, the fill factor (FF) i.e. the maximum power in the current-voltage curve divided by the product of the short-circuit photovoltaic current and the opencircuit photovoltage is an important parameter as it characterizes the quality of the solar cell. Only few papers on photoferroelectrics $[13,74]$ have reported FF data and showed rather weak values of about 10\%-30\% while in classical semiconductors it reaches typically up to $80 \%$. Low FF values are usually attributed to resistive losses via for instance electrodes contact or defects. Such parasitic resistances in photoferroelectrics do also exist and explain partially the low FF values. One may also consider other extra resistances arising from the grain boundaries as well as the domain walls which exhibit different resistance with respect to the bulk. This expectation requires further investigation to reveal properly the parameters controlling the FF.

Concurrently, other fields have been developping among the vast domain of ferroelectrics, such as photostriction or photocatalysis, i.e. the coupling of light with strain or chemistry, respectively. Regarding photovoltaics, potential applications of photoferroelectrics lie in the field of energy harvesting, computing memories, opto-mechanical devices, etc. 


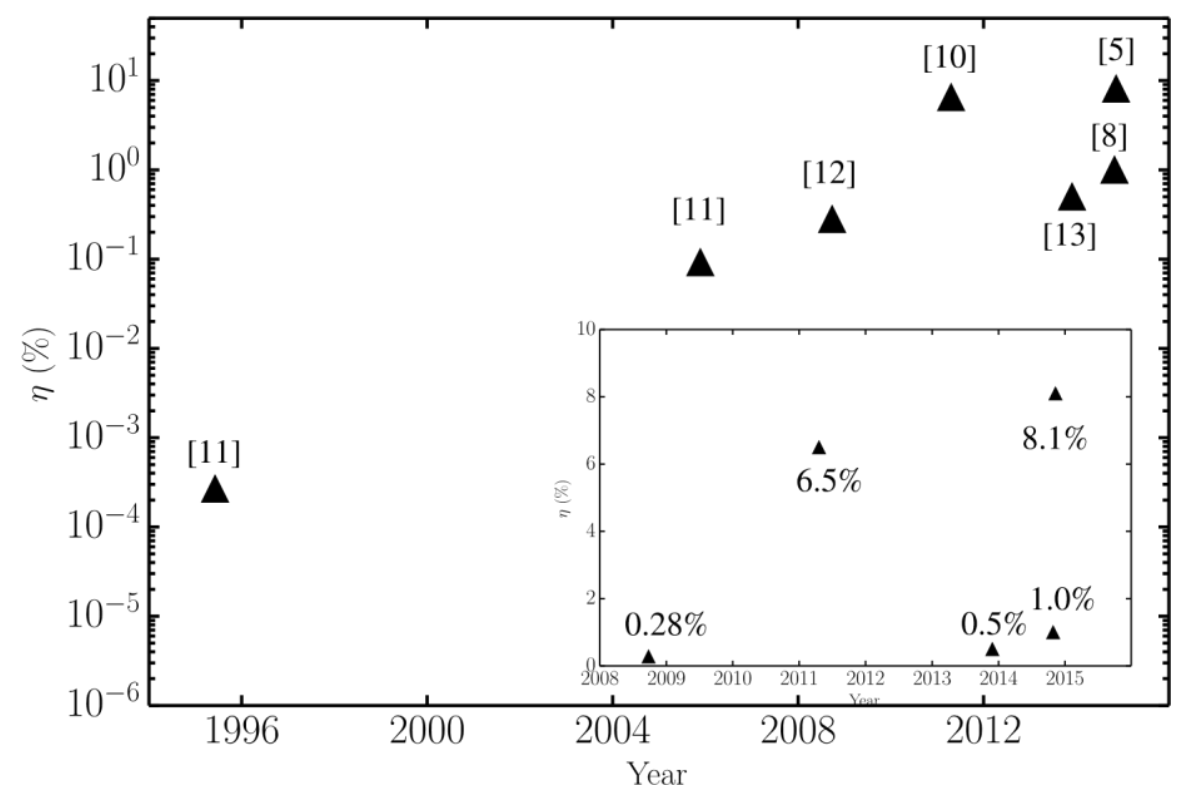

Figure 1: Power conversion efficiency, on a logarithmic scale, over the years from Refs. $[8,11,13,14,15,16]$. The inset shows the most recent achievements on a linear scale.

\section{$2 \quad$ Photovoltaic ferroelectrics}

\subsection{From the 50's to nowadays: a brief review of themodels and controversies}

The first observation of a photovoltaic effect in a ferroelectric dates back to 1956 when the existence in $\mathrm{BaTiO}_{3}$ of a weak pyroelectric current, as well as a steady photovoltaic current above the Curie temperature were shown [17]. These phenomena were attributed to the persistence of a surface charged layer, trapped after heating above the Curie point. It is only in the 1970's that the first quantitative estimates as well as the first models of the photovoltaic effect in ferroelectrics emerged. Pioneering works in 1972 [18] on $\mathrm{BaTiO}_{3}$, and then on $\mathrm{LiNbO}_{3}$ doped with $\mathrm{Fe}^{2+}[19]$ showed the existence of a steady photovoltaic current (electric current flowing in a short-circuited sample under illumination). Even after 20 hours of illumination, a photovoltaic current of $1 \mathrm{nA} / \mathrm{cm}^{2}$ was still recorded in $\mathrm{Fe}^{2+}$-doped $\mathrm{LiNbO}_{3}$. However the most remarkable property is the observation of a photovoltage (voltage under open-circuit condition) of $1000 \mathrm{~V}$ 
under an illumination of $50 \mathrm{~mW} / \mathrm{cm}^{2}$ at a wavelength of $427.2 \mathrm{~nm}$ [19]. In parallel to these experimental observations, the first models appeared. To explain the photovoltaic current in $\mathrm{Fe}^{2+}$-doped $\mathrm{LiNbO}_{3}$, Glass et al. [20] proposed an asymmetric scattering of excited electrons by iron impurities (see Figure 2). Iron impurities in $\mathrm{LiNbO}_{3}$ occupy non-centrosymmetric sites, hence the potential barrier for electrons is not the same in all directions. As one direction is clearly favored, from a probabilistic point of view there will be more electrons with a definite momentum vector $p$. This imbalance results in a non-vanishing photovoltaic current. As there are more photoexcited electrons, the imbalance gets stronger, and so is the photovoltaic current. In addition to the asymmetric scattering of free electrons, the Franck-Condon relaxation of excited ions plays a role in the existence of the photovoltaic current. Finally, the Glass model results in a short-circuit photovoltaic current $J_{s c}$ proportional to the intensity $I$ of the monochromatic incident light $[20,21]$ :

$$
J_{s c}=\kappa_{1} \alpha I
$$

$\alpha$ is the absorption coefficient ( $38 \mathrm{~cm}^{-1}$ in iron-doped lithium niobate) and $\kappa_{1}$ is a constant that depends on the nature and concentration of the impurity center, the mean free path of the excited electron and the energy $\hbar \omega_{0}$ of the incident photons. The Glass model was further refined using a fully quantum calculation of scattering of excited electrons by an asymmetric quantum well [22]. 


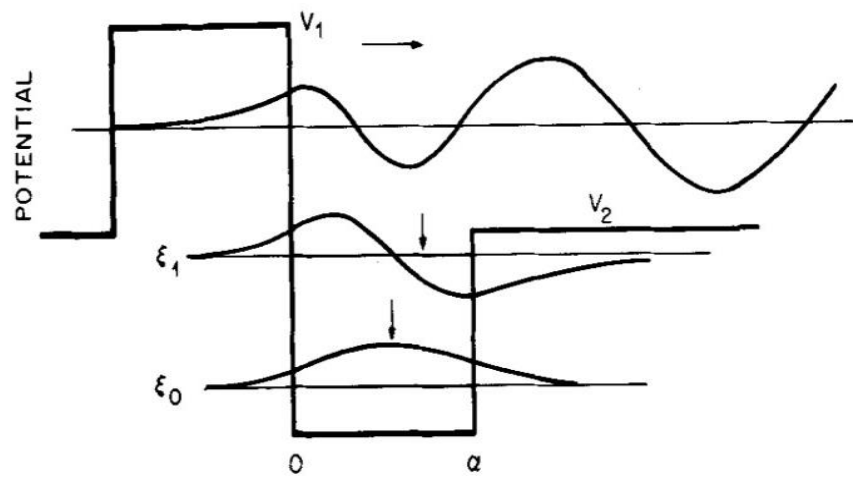

Figure 2: Asymmetric scattering of photoexcited electrons by iron impurities in iron doped lithium niobate. Due to the asymmetry of the barrier, a photoexcited electron has a higher probability to move to the right. Image taken from [20].

However, the Glass model does not account for the sinusoidal variation of the photovoltaic current in Fe: $\mathrm{LiNbO}_{3}$ when rotating the linear polarization of the incident light [7]. To understand this phenomenon, the now-called Bulk Photovoltaic Effect (BPVE) has to be described with a third-rank tensor as a second-order optical response [10, 23, 24, 25]. In a non-centrosymmetric material under an incident electromagnetic plane wave $\boldsymbol{E}_{\text {inc }}$ of frequency $\omega$, wavevector $\boldsymbol{k}$, unit polarization vector $\boldsymbol{e}$, and amplitude $E_{0}$ :

$$
\boldsymbol{E}_{\text {inc }}=\boldsymbol{e} E_{0} e^{i(\boldsymbol{k} \cdot \boldsymbol{r}-\omega t)}+\boldsymbol{e}^{*} E_{0} e^{-i(\boldsymbol{k} \cdot \boldsymbol{r}-\omega t)},
$$

the current density $\boldsymbol{j}$ can be expressed as a Taylor expansion in both the applied electric field $E_{e x t}$ and the incident light electric field having intensity $I=$ $E_{\text {inc }}^{*}, E_{\text {inc }}[23]:$

$$
\begin{gathered}
j_{i}=\sigma_{i j} E_{j}^{\text {ext }}+\sigma_{i j l} E_{j}^{\text {ext }} E_{l}^{e x t}+\sigma_{i j l m} E_{j}^{\text {ext }} e_{l} e_{m}^{*} I+ \\
\chi_{i j l m} q_{j} e_{l} e_{m}^{*} I+\beta_{i j l} e_{j} e_{l}^{*} I+\cdots
\end{gathered}
$$

The first term is the basic Ohm's response to an applied electric field, described by the conductivity tensor $\sigma_{i j}$. The second term is the non-linear 
correction to Ohm's law, described by the tensor $\sigma_{i j l}$. The third term represents the photoconductivity effect, with photoconductivity tensor $\sigma_{i j l m}$. The fourth term is what is called the light entrainment effect, and is usually neglected. The last term represents the Bulk Photovoltaic Effect (BPVE). As the current density is a real quantity, but the photovoltaic tensor $\beta_{i j l}$ is complex, one can separate $\beta_{i j l}$ in real and imaginary parts. The real part $\beta^{L}$ is associated with the linear photovoltaic effect (LPVE) due to linearly polarized incident light, while the imaginary part $\beta^{C}$ describes the circular photovoltaic effect (CPVE) due to circularly polarized incident light. The short-circuit current $j_{s c}$ due to the BPVE can then be written as:

$$
j_{i}^{s c}=\beta_{i j l}^{L} e_{j} e_{l}^{*} I+i \beta_{i l}^{C}\left[\boldsymbol{e} \times \boldsymbol{e}^{*}\right]_{l} I
$$

As the BPVE is described by a third-rank tensor, it can only exist in materials lacking inversion center. According to Ref. [25], materials from any of the 21 piezoelectric crystal classes may exhibit a BPVE effect. Experimentally, a wide variety of such materials have been proved experimentally to exhibit a BPVE, including non-ferroelectric materials such as $\mathrm{GaP}, \mathrm{Te}, \mathrm{ZnO}$ or $\mathrm{HgS}$ [26].

The BPVE photovoltaic current has been historically and phenomenologically expressed in terms of microscopic quantities as [24]:

$$
j_{s c}=e \alpha(\omega)\left(\frac{I}{\hbar \omega}\right) \xi(\omega),
$$

with $e$ being the elementary charge of the electron, $\omega$ the incident light pulsation, and $\xi(\omega)$ the typical mean free path traveled by coherent carriers in a favored direction, i.e. is a typical measure of how far non-thermalized/ballistic carriers will travel in a given anisotropy direction. Such expression has been given further theoretical ground with the development of the so-called Shift Current theory of the BPVE, in which the BPVE tensor can be expressed as the product of a transition intensity (proportional to the absorption) between the $i$-th and $j$-th directions of incident light, $I_{i j}$, and a shift vector in the $l$-th direction, $R_{k}$ [27]: 


$$
\beta_{i j l}^{L}(\omega)=e \sum_{n \prime, n \prime \prime} \int d \boldsymbol{k} I_{i j}\left(n^{\prime}, n^{\prime \prime}, \boldsymbol{k}, \omega\right) R_{l}\left(n^{\prime}, n^{\prime \prime}, \boldsymbol{k}\right)
$$

The summation over $n^{\prime}$ and $n^{\prime \prime}$ has to be understood as a summation over the different bands, while summation over $\boldsymbol{k}$ extends over the whole Brillouin zone. Eq. (6) shows that there are various ways one could develop the necessary asymmetry in $k$-space to have a significant photovoltaic current. Among others, the summation over the Brillouin zone can be asymmetric, especially whenever spin degrees of freedom are involved due to breaking of time inversion symmetry. As a matter of fact, spin currents have been proposed to emerge in antiferromagnets [28]. Also, the shift vector can exhibit anisotropy in $k$-space, one of the easiest representation being the above-presented Glass model, in which the states associated with an asymmetric potential well are scattered differently depending on their wavevector $\boldsymbol{k}$.

The photovoltaic effect in ferroelectrics has remained at a standstill during two or three decades, until 2009 when intriguing results were obtained on bismuth ferrite $\mathrm{BiFeO}_{3}$ (BFO) single crystals exhibiting a switchable diode behaviour [6] and on BFO thin films having a periodic array of $71^{\circ}$ domain walls (see Figure 3 and Ref. [29]). By illuminating these thin films samples with a $285 \mathrm{~W} / \mathrm{m}^{2}$ white light, an above band-gap photovoltage $\left(V_{o c} \approx 16 \mathrm{~V}\right)$ and an in-plane photovoltaic current (of roughly $120 \mu \mathrm{A} \mathrm{cm}^{-2}$ ) flowing against the net global polarization were measured. More importantly, the photovoltage scales linearly with the spacing of the electrode, i.e. with the number of domain walls. In addition, no photovoltaic effect was measured in single domain BFO samples, a fact already stressed out [30] for defect-free samples. Eventually, only the configuration with electrodes parallel to the domain walls (PLDW) exhibits a photovoltaic effect, while the PPDW (electrodes perpendicular to the domain walls) shows none (see Figure 3). Such findings triggered the revival of an active research on photoferroelectrics [31] as well as on domain wall electronics [32]. It is worth mentioning that domain walls for their photovoltaic properties have been investigated mainly in 
BFO while in other ferroelectrics like $\mathrm{BaTiO}_{3}$ (BTO) or $\mathrm{PbZrTiO}_{3}$ (PZT) the research activity focused mostly on their conductive properties [33, 34].
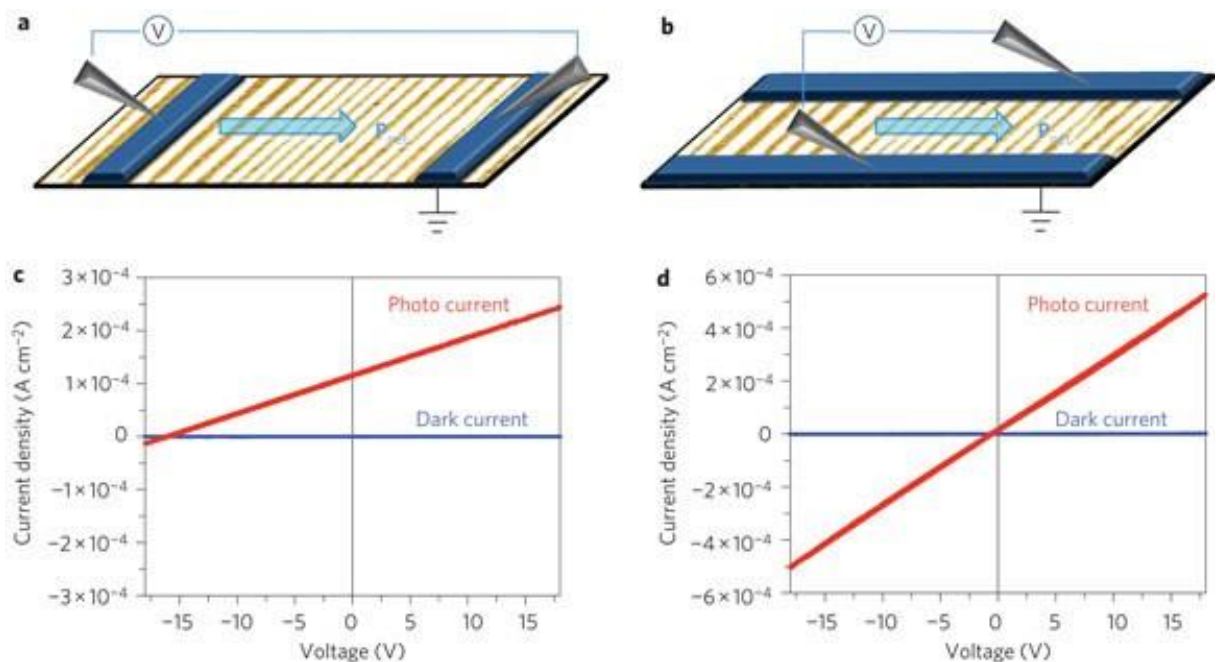

Figure 3: V-I measurement curves for $\mathrm{BiFeO3}$ thin films both in the dark and under 285 W.m-2 white light illumination (a)\&(c) : Parallel to domain wall experiment geometry in which a photovoltaic current is measured. (b)\&(d) : Perpendicular to domain wall geometr geometry : no photovoltaic current is measured. Taken from [29]

To explain the domain wall photovoltaic effect (DWPVE), the following mechanism was proposed. A drop of potential occurs across a domain wall in $\mathrm{BiFeO}_{3}$, mainly due to rigid rotation of the polarization [35]. The drop is largest for $180^{\circ} \mathrm{DWs}$, then for $109^{\circ} \mathrm{DWs}$ (for which photovoltaic effects have also been evidenced [36]), and the smallest drop of potential is given for $71^{\circ} \mathrm{DWs}$. Based on this, a model $[29,37]$ was built on the following assumption: the drop of potential creates a very high electric field in the DW, which thereby separates very efficiently electrons from holes. On the other hand, there is significant recombination in the domain because the internal electric field is not strong enough. Hence, carriers accumulate on different sides of the DW, from which they diffuse, in total analogy to a p-n junction.

However, the DWPVE model has raised controversies and has since then been challenged in many ways. First of all, subsequent first-principle calculations of BFO domain walls could not reproduce the originally predicted bandgap 
variations at the walls [38]. Besides, the model is based on one main assumption: there is a massive recombination in the domains because the electric field is not strong enough to dissociate the photogenerated electron-hole pair. To check the validity of this model, an Atomic Force Microscope (AFM) has been used to probe the local photoelectric effect on a single crystal of $\mathrm{BiFeO}_{3}$ [39]. The $I-V$ curves show no major differences wherever the AFM tip is placed, i.e. on domains or domain walls. Quantitatively similar results are obtained using a macroscopic Pt electrode. In addition, no massive recombination was found in BFO domains [40], and carriers are rather uniformly photogenerated across the entire sample. This study also provides evidence for rather long generation and recombination times of $35 \mu \mathrm{s}$ and $75 \mu \mathrm{s}$, likely due to in-gap defect states, which correlates well with the fact that no clear PV effect was evidenced in good quality single domain BFO crystals [30].

Further proof that the PV effect originated from a BPVE was given by investigating (111)-BFO thin films grown onto (111)-STO substrate [41]. Two gold electrodes were deposited on top of BFO, hence making two symmetric electrodes, ruling out any PV effect arising from asymmetric Schottky barriers. A short-circuit photovoltaic current density [41] of $2 \mu \mathrm{A} . \mathrm{cm}^{-2}$ has been recorded to flow perpendicular to the polarization; a fact that agrees with the tensor nature of the BPVE as expressed in Refs. $[23,10]$ or Equation (4). In addition, a sinusoidal modulation of the photovoltaic current was observed when varying the polarization direction of the incident light, a clear signature of the BPVE, as also shown in $[6,42]$.

The role of domain walls has been elucidated only recently through the study of the photovoltaic, photoconductive and conductive properties of $109^{\circ}$ and $71^{\circ}$ DWs arrays in BFO as a function of the temperature [42]. Symmetric Pt electrodes were used to avoid contributions from asymmetric contacts. At room temperature, a high photovoltage of $7 \mathrm{~V}$ was found for $71^{\circ} \mathrm{DWs}$ in PPDW and PLDW geometry, while for $109^{\circ} \mathrm{DWs}$, a photovoltage of $5.4 \mathrm{~V}$ was recorded in the 
PLDW set up, but none in the PPDW geometry. This is at variance with the proposed DW model $[29,37]$. In addition, the open-circuit voltage $V_{o c}$ decreases exponentially as the temperature is increased, regardless of the geometry of the setup. Concurrently, both the conductivity and the photoconductivity increase exponentially. Assuming an almost constant photovoltaic current, the photovoltage is related to the conductivity $\sigma$ and to the photoconductivity $\sigma_{p h}$ through:

$$
V_{o c}=\frac{L}{\sigma+\sigma_{p h}}
$$

with $L$ being the spacing between the electrodes. This relation is consistent with the exponential decrease of the photovoltage with temperature. The role of the domain wall is limited to modulating the global conductivity. In the PLDW geometry, they act as extra-resistances that add to the bulk resistance, and in the PPDW geometry, they act as shunts, due to the fact that the domain walls in $\mathrm{BiFeO}_{3}$ are more conducting than the bulk [43, 44, 45] (see Figure 4).

Eventually, it has been shown [42] that upon rotating the direction of polarization of the light, a variation of the photovoltaic current (see Figure 5) is observed which is fully consistent with the tensor model of the BPVE as developped by Fridkin et al. [10, 23]. Differences in the photovoltaic current depending on the polarization of light had already been evidenced in BFO [6] as well as in early photoferroelectrics such as lithium niobate $[7,46]$. This is further confirmed by the comparison to the so-called shift current model $[24,25]$, a theory that was later implemented in Density Functional Theory (DFT) calculation to predict the BPVE tensor $[27,47]$ in BFO. 


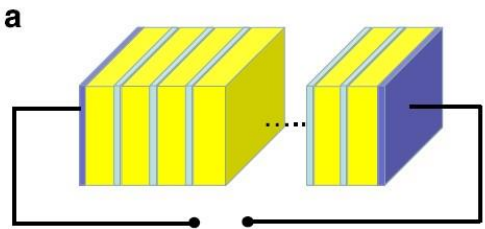

a

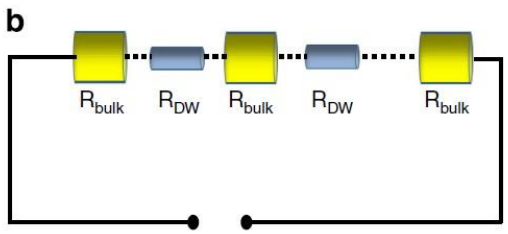

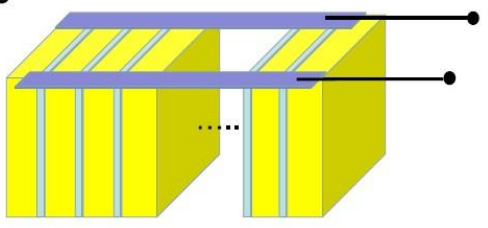

d

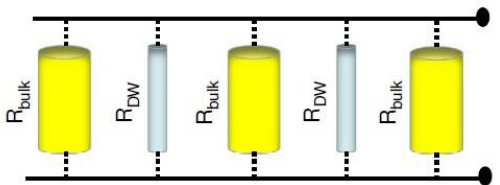

Figure 4: (a) \& (b) PLDW geometry set up and analogous electrical circuit. (c) \& (d): PPDW geometry set up. Taken from [42].

In conclusion of this section, from the latest experimental data available, there exists a photovoltaic effect in ferroelectric materials that is intrinsic, and of tensorial nature: this is the so-called Bulk Photovoltaic Effect. However, in addition to domain walls the photovoltaic effect in ferroelectrics can be altered/tailored by the use of extrinsic and external parameters, such as an electric field, electrodes or defects.

\subsection{Tailoring the photovoltaic effect: towards real-life}

\subsubsection{Switchability}

Just as a regular $p-n$ junction, thin films ferroelectrics like bismuth ferrite exhibit a diode behaviour $[6,48,49,50,51]$. More importantly, the diode forward direction can be controlled by the polarization direction, as shown on BFO single domain samples contacted with gold or silver symmetric electrodes, in which the diode forward direction was switched by reversing of the polarization [6]. Additionally, a steady photovoltaic current was observed under green light illumination in those BFO samples. These two observations naturally lead to wonder whether the switchability property of the diode applies also to the photovoltaic current. 


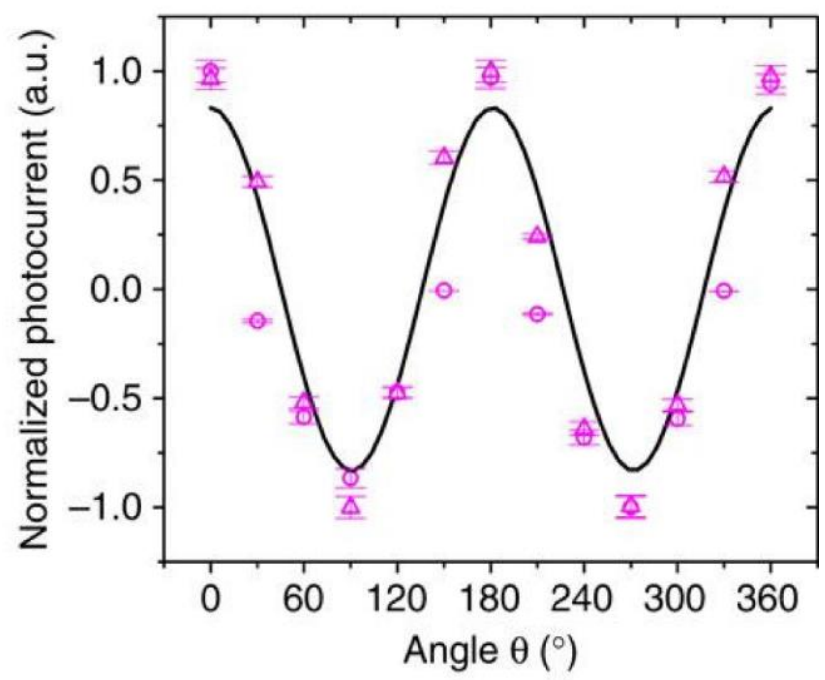

Figure 5: Variation of the photovoltaic current in BFO thin films of $71^{\circ} \mathrm{DWs}$ (triangles) and $109^{\circ} \mathrm{DWs}$ (circles). The plain line is the fit with the BPVE model. Extracted from [42]. 

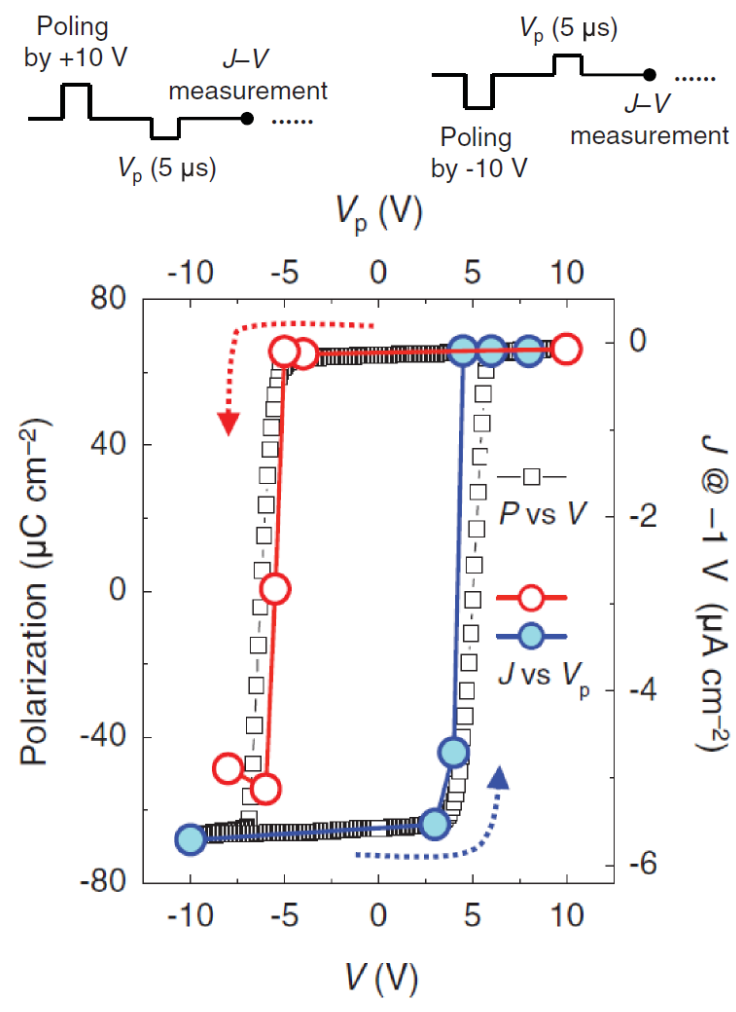

Figure 6: The photovoltaic current direction follows the same hysteresis loop as the polarization in Pt/BFO/SRO thin films. Reproduced with permission from [52]. Copyrighted by the American Physical Society.

Perhaps the most striking answer to that question was brought by Lee et al. [52]. Using a Metal-Ferroelectric-Metal (MFM) device consisting of a $400 \mathrm{~nm}$ thick BFO film sandwiched between a Pt and a single crystal SRO electrodes, they demonstrated that the photovoltaic current across the MFM structure perfectly superimposes onto the polarization hysteresis loop (see Figure 6).

It turns out that several other reports show that the photovoltaic current can be switched with the polarization as well, for instance in $\mathrm{BFO}[53,52]$ or $\mathrm{WO}_{3}$-doped PLZT [54], or at least tailored by the polarization [12]. As an example of how the switchable property of the BPVE can be used towards real-life applications, a 100 nm thick BFO film sandwiched between $\mathrm{La}_{0.7} \mathrm{Sr}_{0.3} \mathrm{MnO}_{3}$ and $\mathrm{Pt} / \mathrm{Fe}$ electrodes revealed that a reversible short-circuit photovoltaic current and open-circuit voltage of $0.15 \mathrm{pA} /-0.13 \mathrm{~V}$ for down polarization and $-0.15 \mathrm{pA} / 0.21 \mathrm{~V}$ for up 
polarization can be obtained [55]. The asymmetry is likely to come from the different chemical nature of the two electrodes, an issue to which we shall return later on. Taking advantage of the switchable property allowed to design a 16 cells prototype of a photoferroelectric memory [55]. A similar photovoltaic current reversal was observed in a $400 \mathrm{~nm}$ thick $\mathrm{BFO}$ film in a $\mathrm{Pt} / \mathrm{BFO} / \mathrm{SrRuO}_{3}$ architecture [52]. The photovoltaic current density of $0.1 \mu \mathrm{A} / \mathrm{cm}^{2}$ under white light illumination of less than $10 \mathrm{~mW} / \mathrm{cm}^{2}$ reverses upon switching of the polarization (see Figure 7). Interestingly, as the polarization state of the sample is probed with PiezoForce Microscope (PFM), no photovoltaic current was detected when the sample is in an equally mixed state of up and down polarization (see Figure 7 and Ref. [52]).

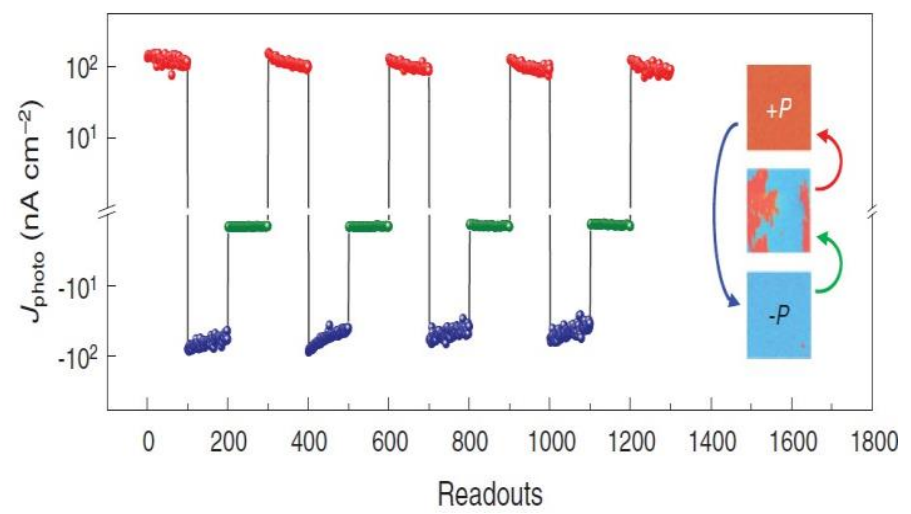

Figure 7 : Measured photovoltaic current in Pt/BFO/SRO thin films as a function of the polarization state, as seen by PFM. In the down state (blue), the photovoltaic current is opposite to the the photovoltaic current in the up polarization state (red). In the mixe mixed state, no photovoltaic current was detected. Reproduced with permission from [52]. Copyrighted by the American Physical Society.

The switchability of the photovoltaic current is fully consistent with the BPVE model, as reversing the polarization accounts for performing spatial inversion. Since the BPVE tensor is odd, the photovoltaic current should change sign, but 
with equal magnitude, thereby explaining both the switchability property and that on average, the photovoltaic current in an equally mixed state of up and down domains macroscopically vanishes. Note also that the photovoltaic current vanishes above the Curie temperature in BTO [56, 46] and PZT [57], when spatial inversion symmetry is recovered in the high symmetry paraelectric phase.

If switchability is of great interest for potential applications as photoferroelectric memories, it is not always achievable because of extrinsic parameters such as the nature of the electrode-ferroelectric interface (Schottky barrier) or the quantity of non-mobile charged defects.

\subsubsection{Electrodes: is there a better way to integrate a photoferroelectric?}

Because electrodes form Schottky barrier at the interface with a ferroelectric [58, $59,60,61]$, they can easily be either detrimental to transport properties by creating high contact resistance, or advantageous in the design of ferroelectric diodes if only one interface is a Schottky contact, while the other has an ohmic character. Thus, careful attention must be paid to the choice of contacting electrodes in order to achieve efficient and/or switchable photoferroelectric devices. The advantage of using ferroelectrics instead of classic semiconductors stems from the possibility of tuning the barrier height of the Schottky contact by playing with the polarization. Indeed, the electric polarization alters the Schottky barrier at the interface, modulating the barrier by the amount

$$
\Delta \Phi_{P}= \pm \frac{P}{\epsilon_{0} \epsilon_{r}} \delta,
$$

depending whether the polarization $P$ is pointing towards the electrode $(-)$ or away $(+)[59,60] . \epsilon_{r}$ is the dielectric constant of the ferroelectric and $\delta$ the spatial extent of the interfacial layer. For [001]-oriented BFO films, the barrier change has been estimated to be of the order of $\Delta \Phi_{B F 0} \approx \pm 0.6 \mathrm{eV}$ [52]. The choice of electrodes is critical when one wants to achieve switchabil ity of the photovoltaic effect, to design photoferroelectric memories for instance [51]. 
Indeed, devices exhibiting high Schottky barrier heights compared to the polarization-induced change $\Delta \Phi_{P}$ are unlikely to be switchable. As an example, Yang et al. epitaxially grew $\mathrm{BFO}$ thin films on $\mathrm{SrRuO}_{3}$ (SRO), with Indium Tin Oxide (ITO) as a semi-transparent top electrode [62]. For [001]-oriented BFO thin films, no difference in their diode and PV characteristics is observed whether the polarization is up or down. On the other hand, for [111]-oriented films, for which the number of polarization charges at the interface with the electrode is $\sqrt{3}$ larger, a significant change of one order of magnitude in the short-circuit photovoltaic current is evidenced upon switching the polarization. The barrier height between ITO and BFO was then estimated to be roughly $1.25 \mathrm{eV}$, which is larger than the barrier change $2 \times \Delta \Phi_{B F O}$ that can be induced by switching the polarization in a [001]-oriented film, but smaller than the barrier change $2 \times \sqrt{3} \times \triangle \Phi B F O$ induced by a [111]-oriented BFO film.

An extensive study of the top electrode effect was conducted on a PZT (20/80) thin film deposited on a SRO bottom electrode on top of a STO(100) substrate [63]. The authors observed that the switching behavior does not occur for every electrode, but only for Pd, Ag or SRO top electrodes. In other cases, such as $\mathrm{Cr}$, Pt or $\mathrm{Al}$, no reversal of the photovoltaic current was observed when switching the polarization (see Figure 8). To account for the different behavior with different top electrodes, the author proposed a similar mechanism of modulation of the space charge region at the metal-ferroelectric interface, but a quick estimate ruled out this possibility.

The fundamental understanding of the impact of electrodes and interfaces on photovoltaic properties of ferroelectrics still remains an open question, as the modulation in Schottky barrier may also be caused by defective layers of oxygen vacancies [52], and other photo-induced effects at the interface can occur, for instance the emission of photoelectric electrons from the electrode. As a matter of fact, Zhang et al. studied the effect of three different metallic bottom electrodes 
(Ag, pt or Mg) on a ITO/PLZT(100 $\mu$ m thick pellet)/Metal capacitor-like geometry [64], using light with a spectral distribution mainly below the bandgap of PLZT (see Table 1). They observe that the lower the work function of the metallic electrode, the higher was the short-circuit photovoltaic current and the opencircuit photovoltage. As a result, the ITO/PLZT/Mg sample (with estimated Shottky barrier $\Phi_{B}=0.2 \mathrm{eV}$ ) has a photovoltaic current and a photovoltage 150 and 2 times as large as the ITO/PLZT/Pt ( $\Phi_{B}=2.0 \mathrm{eV}$ ). In addition, when shining light on the metallic interface, i.e. light does not penetrate the PLZT, non-negligible photovoltaic current and photovoltage are still measured. They concluded that significant photoelectric effect from the metallic electrode happened, and can enhance the photovoltaic effect due to PLZT and interface Schottky barriers, pointing at the fact that a system based (at the scale of a complete solar cell) and not only a single material based approach is necessary in the quest for high PCEs photoferroelectric devices.

As a result, the PV characteristics of ferroelectric materials can be tailored by the careful choice of electrodes. As another example, an enhancement of one order of magnitude of the PCE in PLZT was obtained by using Al instead of Pt as a top electrode in an Al/PLZT/Pt device [65]. Numerical simulations also demonstrated that among metallic electrodes, those with lowest dielectric constant are more suitable to enhance the diode switchability properties of ferroelectrics, as the change in Schottky barrier height with polarization is 5 times as large for the $\mathrm{Pt} / \mathrm{BFO}$ interface when compared to a SRO/BFO interface [49]. On the other hand, other calculations predict that oxide electrodes such as ( $\mathrm{La}, \mathrm{Sr}) \mathrm{MnO}_{3}$ (LSMO) with high dielectric constant would yield dramatic improvements in the PCE (up to $19.5 \%$ !) of PLZT [12]. It is to remind that none of these simulations take into account the BPVE effect, as they are based on the resolution of the Poisson equation, and classical transport equations. Experimentally, the photovoltaic response was shown to be higher in Pt/BFO/LSMO structure than in LSMO/BFO/LSMO structure [66]. 

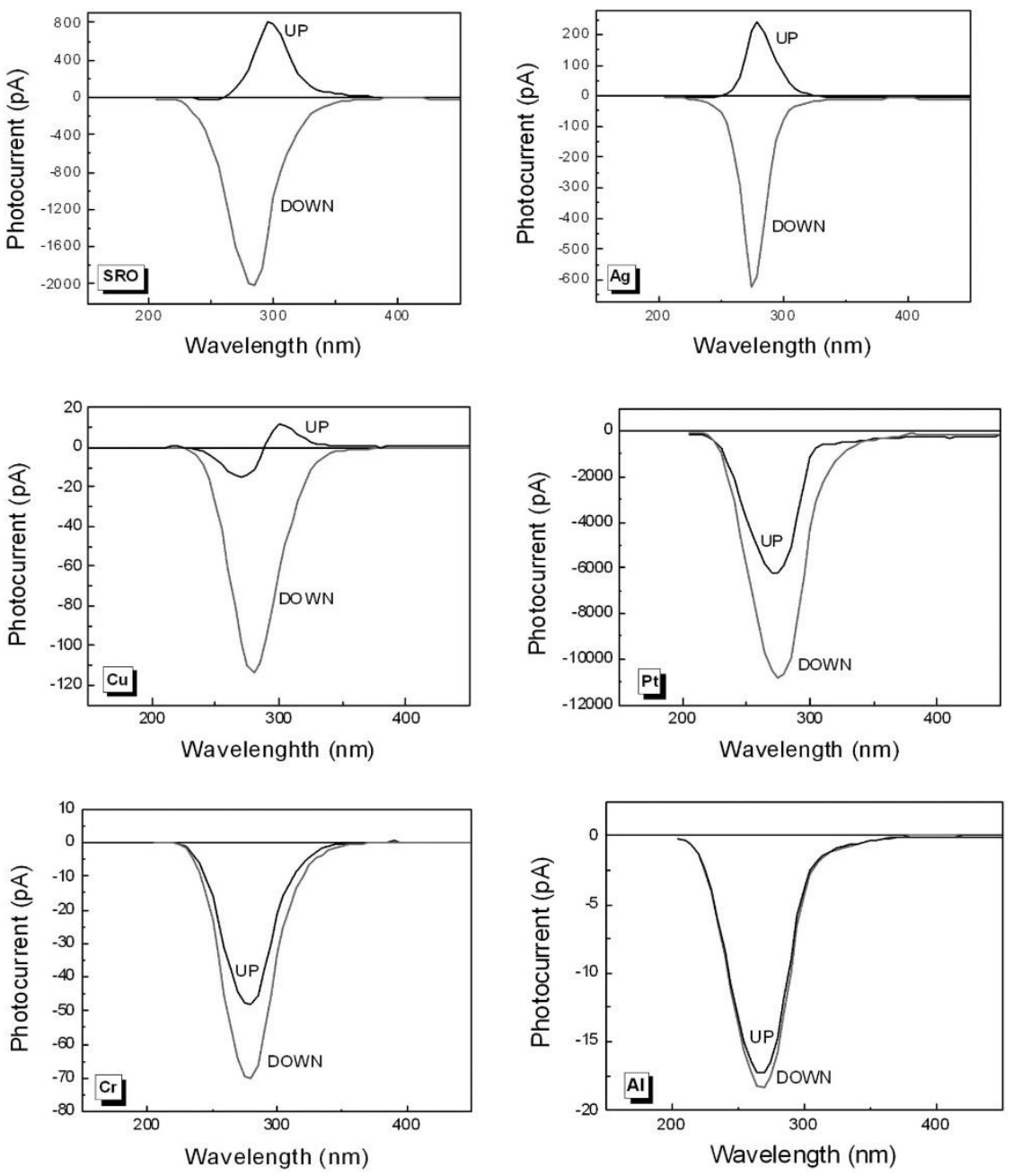

Figure 8: Measured photovoltaic current for different top electrodes on a Metal/PZT/SRO/STO(100) thin film in a capacitor-like geometry with an SRO bottom electrode. Extracted from [63].

For potential applications in photoferroelectric memories, it is interesting to systematically estimate how switchable the photovoltaic current is, by calculating what we name the switchability of the device, $\frac{j_{u p}-j_{\text {down }}}{2 j_{u p}}$, with $j_{u p}$ being the photovoltaic current density in the up polarization state of a uniaxial ferroelectric, and $j_{\text {down }}$ the photovoltaic current in the down state. It was estimated to be of the order of $70 \%$ in $\mathrm{Bi}_{2} \mathrm{FeCrO}_{6}$ films on $\mathrm{SrTiO}_{3}: \mathrm{Nb}$ substrate with ITO top electrodes [13]. The switchability is a rough estimate of the bulk-controlled part of the photovoltaic effect when the temperature is low enough compared to the energy 
barrier of diffusion of oxygen vacancies. Indeed, the latter have been demonstrated to create strong internal fields at the interface with the electrodes, and their possible migration from one electrode to the other can also contribute to the switchability of the photovoltaic current, as we shall see in the next section $[52,67]$. Causes for low switchability are often the presence of strong internal fields at interfaces, due to accumulation of non-mobile defects or very high Schottky barrier.

\subsubsection{Defects: friend or foe?}

Just as the electrodes create built-in fields in a ferroelectric, so do defects as oxygen vacancies. They can critically affect the PV properties of ferroelectrics, as for instance switchability of the photovoltaic and diode properties of $\mathrm{Pt}$ /BFO/SRO thin films could not be achieved until removing oxygen vacancies away from the electrode by applying a bias higher than the coercive field [52] Similar observations were made on BFO crystals contacted with gold electrodes in a capacitor-like geometry [67].

The role of oxygen vacancies was further highlighted [51]. Both a BFO and a $\mathrm{Bi}_{0.9} \mathrm{Sr}_{0.1} \mathrm{FeO}_{3-\delta}$ (BSFO) polycrystalline thin film, deposited on fluorine-doped tin oxide (FTO) with a gold top electrode, were studied. It is well known that oxygen vacancies are present in BSFO in a larger quantity than BFO, but transmittance measurement showed a similar band gap of $2.75 \mathrm{eV}$ for both materials. Applying voltages $(-2.5 \mathrm{~V}$ and $+2.5 \mathrm{~V})$ below the coercive field allows to reverse the photovoltaic current from $50 \mu \mathrm{A} / \mathrm{cm}^{2}$ to $-20 \mu \mathrm{A} / \mathrm{cm}^{2}$ on a time scale of $20 \mathrm{~min}$ in BSFO, while no significant change of the photovoltaic current was observed in BFO. Now, poling at voltages larger than the coercive field $( \pm 10 \mathrm{~V})$ modified significantly the photovoltaic current and diode behavior in both samples. It was concluded that a competition occurs between the polarization and the space charge layer induced at the ferroelectric-metallic electrode interface [51]. From the latter results, one understands that in defect-free $\mathrm{BFO}$, the photovoltaic effect is mainly driven by the polarization, either the BPVE or modulation of the 
Schottky barriers at the ferroelectric-metal interfaces, while in highly defective BSFO, the photovoltaic effect is mainly driven by the position of the space charge layer of oxygen vacancies, and its effect on band-bending at interfaces.

Far from being only detrimental, oxygen vacancies can actually be helpful, by either creating internal screening fields, aggregating at interfaces, sub-bandgap levels to absorb a broader part of the solar spectrum, or just enhancing the conductivity of a material. No PV effect in a single domain BFO could be detected until they shone intense light on the sample, and concurrently apply an electric field below the coercive field, thereby creating oxygen vacancies that migrate to one of the $\mathrm{BFO} / \mathrm{Au}$ interface [30]. After such treatment, a short-circuit photovoltaic current of $0.6 \mathrm{nA}$ was measured under $50 \mathrm{~W} / \mathrm{cm}^{2}$ white light illumination, which Moubah et al. interpret as the result of band-bending at the interface caused by oxygen vacancies. To further back up their interpretation, they show that the photovoltaic current is stable over $20 \mathrm{~min}$ when shining light over the gold electrode, whereas the photovoltaic current obtained from light shone in-between electrodes decays to zero with a time constant of $26.6 \mathrm{~min}$. Note also that shining light on the gold electrode can also contribute to the photovoltaic current via the photoelectric effect in metals, as pointed out by Zhang et al. [64], and is not discussed by the authors of Ref. [30].

Defects such as oxygen vacancies are obvious sources of modulation of the conductive properties of ferroelectric materials, and are widely believed to make materials suffer from leakage. This leads to a deterioration of the diode properties, illustrated by samples of $\mathrm{BaTiO}_{3} / \mathrm{Si}$ showing lower threshold conduction voltage when synthesized under low oxygen partial pressure (see Figure 9 and Ref. [68]). However, this can possibly enhance the photovoltaic current output. Indeed, upon shining a laser with photons having a higher energy than the band gap of BTO, a photovoltaic response was observed in all BTO samples, but was higher in samples having high oxygen vacancies concentration $\left(V_{o c} \approx 37 \mathrm{mV}\right.$ for high oxygen vacancies concentration samples vs $2 \mathrm{mV}$ for low 
oxygen vacancies concentration samples). On the other hand, illumination with a HeNe laser (with wavelength $632.8 \mathrm{~nm}$, well below the bandgap of $\mathrm{BaTiO}_{3}$ ) shows that only samples grown in oxygen poor atmosphere exhibit a significant photovoltaic response. This was explained by the formation of a $p-n$ junction at the interface between a space charge layer of oxygen vacancies and the $p$-doped silicon. Such mechanism had already been postulated in 2000 to explain the photovoltaic effect in PZT(48/52) [69].

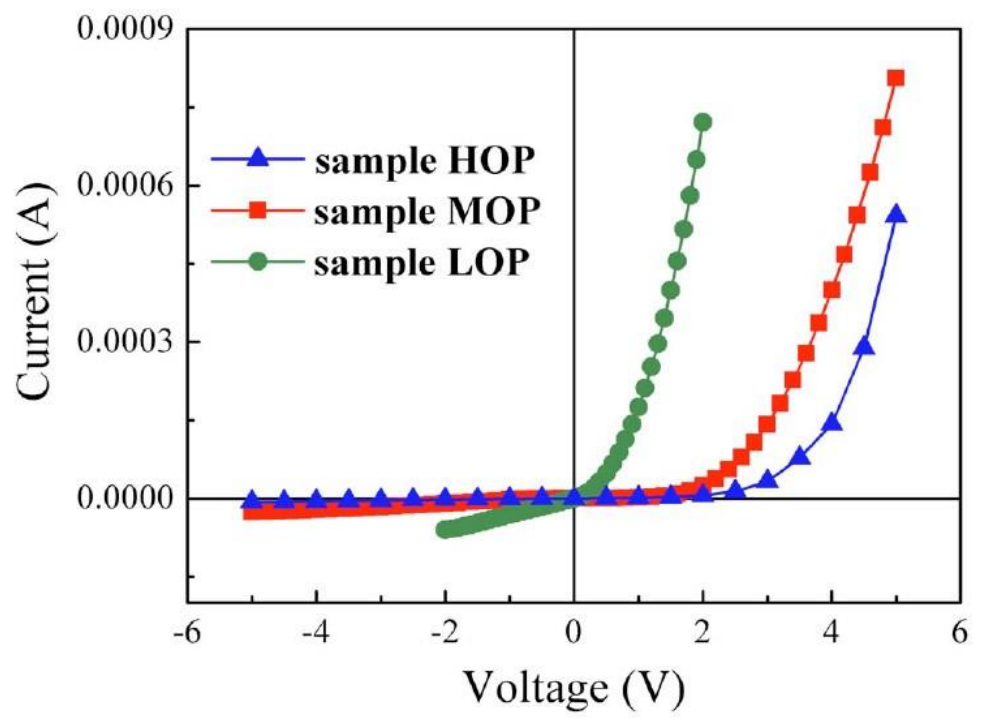

Figure 9: I-V characteristic curves for barium titanate samples fabricated under High/Middle/Low Oxygen Pressure (H/M/LOP). The diode behavior worsens with the increasing oxygen vacancy concentration, while the threshold voltage for rectification decreases. Taken from [68]. 
On a similar note, Yang et al. observed an enhancement by a factor of 2 of the photovoltage and photovoltaic current in $\mathrm{BiFeO}_{3}$ single crystals grown with

a

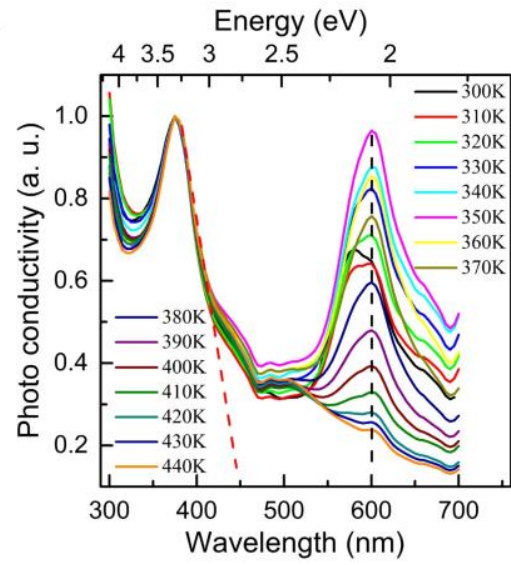

b

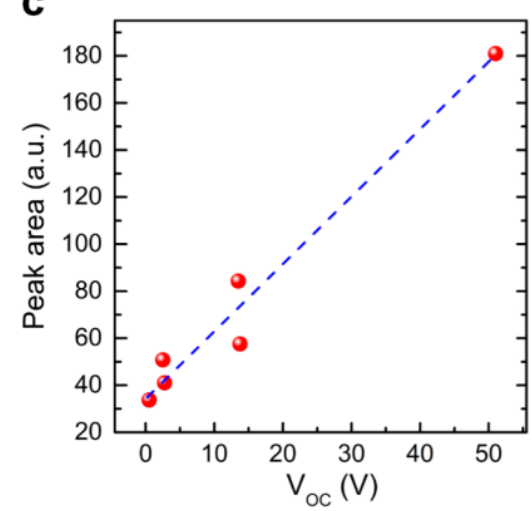

Figure 10: (a) Variation with the temperature of the sub-band response in $\mathrm{BiFeO3}$ single crystal and (b) open circuit voltage as a function of area of the sub-band peak centred at $600 \mathrm{~nm}$ as shown in (a). Extracted from [70].

different orientations, domain patterns, or even different laboratories, by using photons carrying sub-bandgap energy [70]. Such enhancement was attributed to a simple electronic pumping of sub-bandgap levels situated at $2.2 \mathrm{eV}$, as the spectral photoconductive response exhibit an important response in the area between 2.2 and $2.6 \mathrm{eV}$ (see Figure 10.a). In addition, the recorded photovoltage response of the $\mathrm{BiFeO}_{3}$ is directly proportional to the area of the sub-gap photoconductive peak (see Figure 10.b), clearly correlating the PV response of BFO with defect centers when illuminated with sub-bandgap light. It follows that the nature of the defect centers (i.e. whether they act as recombination centers or trapping levels) will affect drastically the BPVE outputs such as the open-circuit bandgap. These results highlight the crucial role of sub-bandgap levels in fulfilling one of the most important condition of existence of BPVE, respectively that the recombination processes must proceed in ways that are different from the carrier excitation processes to create the necessary asymmetry in the reciprocal $k$ space. Up to now, the importance of the defect states and how sensitive can the BPVE be to these electronic details has been largely neglected. 
A fine control of defects, especially oxygen vacancies, appears as a decisive step to either achieve switchability (for potential applications as photoferroelectric memories) or energy harvester (to tailor the PV signal). Once again, disentangling the impact of defect, electrodes or polarization on the PV properties is not an easy task. Previously described experiments however do point to the fact that studying switchability after applying electric fields below the coercive field may give a qualitative estimate of the importance of defects in the PV output. The switchability property of the PV current after applying electric fields both below and above the coercive field can therefore serve as a qualitative (if not quantitative) probe of the dominating mechanisms in ferroelectric-based PV devices.

\subsubsection{Size effects: the smaller the better?}

In the design of photoferroelectrics cells, thin films have been widely investigated. The optimization of their geometry is critical to achieve efficient PV yields in photoferroelectrics. Early reports showed that thinner UV-illuminated $\mathrm{Au} / \mathrm{PLZT} / \mathrm{Pt}$ showed higher photovoltaic currents $U_{s c} \approx 0.85 \mathrm{nA}$ at $260 \mathrm{~nm}$ film thickness under $1.6 \mathrm{~mW} / \mathrm{cm}^{2} \mathrm{UV}$ irradiation). The interplay between the space charge region at the electrode/FE interface and the screening effect of the electrodes play a decisive role in tailoring the PV effect. Moderate efficiency of $0.28 \%$ was achieved experimentally in PLZT thin films [15]. As shown numerically on PLZT films contacted with different electrodes, there exists a critical thickness $L_{o p t}$ that yields the maximum achievable photovoltaic current (see Figure 11) [12]. Lopt is the threshold between a regime in which the photovoltaic current is limited by polarization screening by the electrodes, and a regime (below Lopt) in which the photovoltaic current is commanded by the Schottky-induced space charge region.

An early model [71], based on the Glass model for the PV effect, agrees qualitatively well with the numerical results [15]. The photovoltaic current is 
related to the width $(w)$ and length $(L)$ of the illuminated face, the thickness $t$ of the film by the following relation [71]:

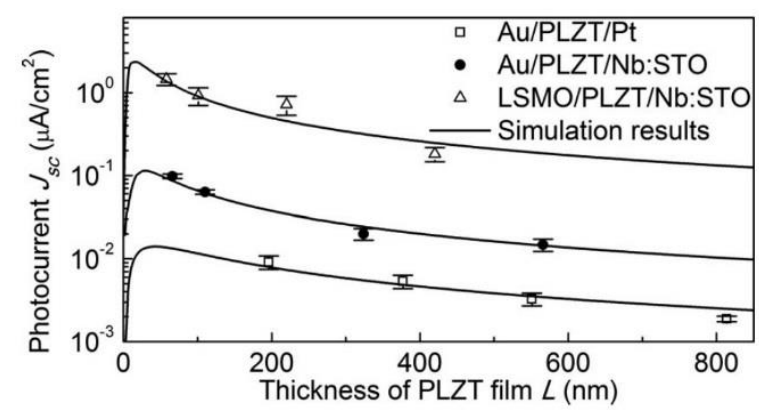

Figure 11: Short-circuit photovoltaic current vs thickness of PLZT thin film, as determined from calculations with different electrodes. There exist a thickness Lpeak for which Jsc is maximum. Extracted from [12].

$$
J_{S c}=\frac{w \kappa T I_{0}\left(1-e^{-\alpha t}\right)}{1+R_{m} w\left(\frac{\sigma_{d} t}{L}+\frac{\sigma_{p h^{T I}}\left(1-e^{-\alpha t}\right)}{\alpha L}\right)}
$$

with $\alpha$ the absorption coefficient of the material, $T$ its transmittance, $I_{0}$ the intensity of the incident light, $\kappa$ the Glass coefficient (see Equation (1)), $\sigma_{d}$ and $\sigma_{p h}$ are the dark conductivity and photoconductivity respectively. With this model, an optimal thickness of $33 \mu \mathrm{m}$ in $\mathrm{WO}_{3}$-doped PLZT films was predicted [71].

In ceramics, the size effect can matter as well. As it is composed of many connected grains which carry an individual photovoltage, a giant photovoltage of several thousands of volts can build up, for instance in PLZT [72, 73]. Therefore, the open-circuit photovoltage increases linearly with the length that separates the two electrodes, and the relevant quantity to look at is the open-circuit photoinduced electric field; $E_{o c}$. Interestingly, the open-circuit photo-induced electric field has been reported to increase with decreasing grain size in PLZT ceramics (see Figure 12 and Ref. [73]).

It is worth noting that very recently, a PCE of $0.6 \%$ has been achieved in $\mathrm{BaTiO}_{3}$ thin films with thickness below $100 \mathrm{~nm}$, which represents an enhancement by a 


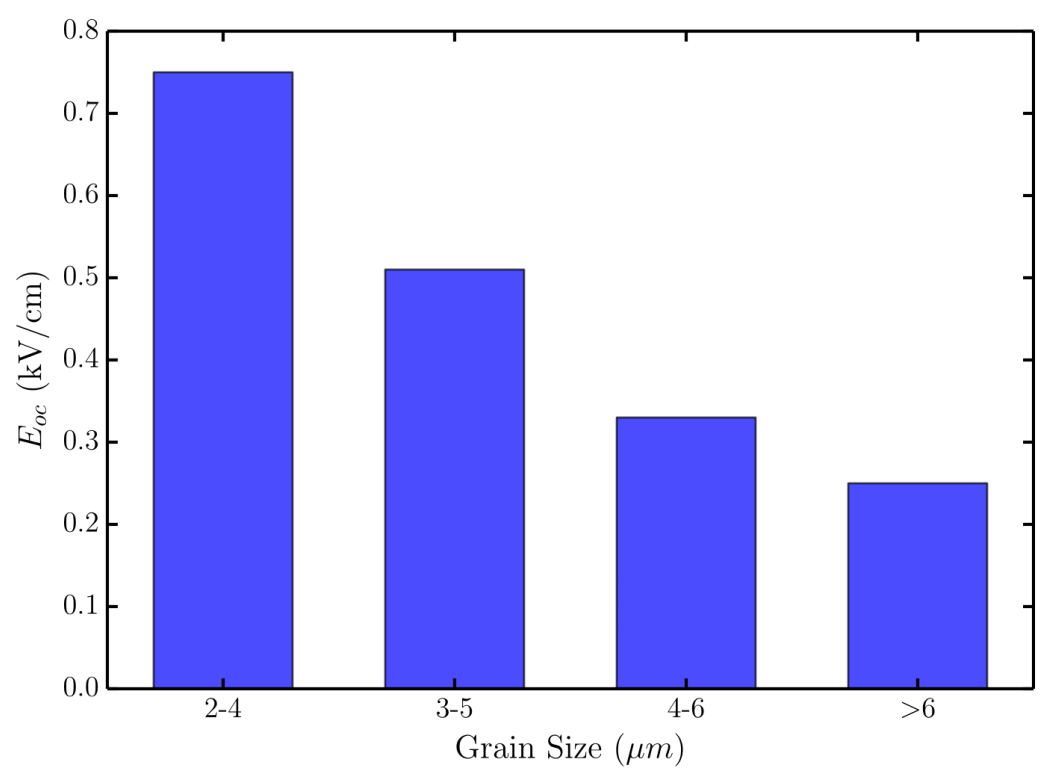

Figure 12: Photo-induced open circuit electric field Eoc with respect to the grain size in PLZT ceramics. Data taken from Ref. [72].

factor of 4.5 of the yield with respect to bulk crystals [11].Such enhancement was made possible by reducing the size of the device below/close to the mean free path of non-thermalized carriers, estimated to be of the order of 10-100 nm, in order to reach a ballistic regime for all excited carriers. In a similar fashion, in $\mathrm{BiFeO}_{3} / \mathrm{BiCrO}_{3}$ bilayers, a high photovoltaic current of $130 \mu \mathrm{A} / \mathrm{cm}^{2}$ has been recorded for $15 \mathrm{~nm}$ thick layers, twice as much as the photovoltaic current measured in $40 \mathrm{~nm}$ thick layers [74].

\subsubsection{Band-gap engineering}

So far, PV effects in photoferroelectrics have been very limited by poor yields, but also by the excessively high band gaps found in most ferroelectrics. With band gap values of typically $3 \mathrm{eV}$ or higher (see Table 1), most of them absorb in the UV range. Being able to tailor their band gap to get a maximum of absorption around $1.5 \mathrm{eV}$, around the maximum of the solar spectrum, is therefore the ultimate goal. Several strategies and guidelines for engineering band gaps in ferroelectrics have been considered. 
First, playing with the structure and the chemical ordering is one way to achieve efficient reduction of the bandgap. In extremely tetragonal perovskite structures such as $\mathrm{Bi}(\mathrm{Zn}, \mathrm{Ti}) \mathrm{O}_{3}$ (BZT), the bandgap has been shown to be very sensitive to local chemical ordering of the $B$-cation. For alternate stacking of planes of $\mathrm{Zn}$ and Ti cations in the tetragonal direction, it has been shown by DFT calculations that a strong decrease of the bandgap (about $0.5 \mathrm{eV}$ ) was achieved as compared to other chemically ordered cation arrangement [75]. This is of interest since the tetragonal structure is commonly obtained in thin films by using strain, and seems to be desired as Wang et al. showed that the band gap of the tetragonal structure of various perovskites was systematically lower than its rhombohedral counterpart [76]. In addition, the author of Ref. [75] showed that suppressing the oxygen octahedra rotation in those tetragonal structures participate to lower the bandgap. Chemical ordering of $\mathrm{Cr}$ and $\mathrm{Fe}$ cations in $\mathrm{Bi}_{2} \mathrm{FeCrO}_{6}$ (BCFO) thin films, as characterized by the intensity and width of superstructure diffraction reflections, was correlated with Jahn-Teller distortions and lowering of the bandgap down to $1.43 \mathrm{eV}$ [8]. The control of the chemical ordering by the deposition rate hence allowed the authors of Ref. [8] to grow a three multilayer of the same material, BFCO, with each layer absorbing a different part of the solar spectrum because of their different chemical ordering. Such design of a multilayered structure to achieve efficient light harvesting yielded the highest efficiency (8.1\%) ever recorded for conventionnal ferroelectrics, with characteristic photovoltaic outputs $J_{s c}=11.7 \mathrm{~mA} / \mathrm{cm}^{2}$ and $V_{o c}=0.79 \mathrm{~V}$ under AM1.5 illumination. Note that an earlier work on $\mathrm{Bi}_{2} \mathrm{FeCrO}_{6}$ thin films already showed a $6.5 \%$ PCE under a red laser, with a photovoltaic current density of $0.99 \mathrm{~mA} / \mathrm{cm}^{2}$, one of the largest ever recorded [13]. It is also interesting to notice that the stacking of light harvester materials with different bandgaps is one of the solution to push the Shockley-Queisser limit of p-n junctions up [77], and that this similar strategy, as employed by Nechache et al. [8], is of large interest in order to absorb as much energy as possible from the solar spectrum. 
Besides control of chemical ordering "at constant chemistry", as examplified by the device designed by Nechache et al., another possible route to lower the bandgap would be to play with chemistry, by using solid solutions or doping. Solid solutions allow to tailor the properties in between two or more end members. In the case of $\left(\mathrm{KNbO}_{3}\right)_{(1-x)}\left(\mathrm{BaNi}_{0} .5 \mathrm{Nb}_{0.5 \mathrm{O}_{3}}\right)_{x}(\mathrm{KBNN})$, which is a mixture of a ferroelectric $\left(\mathrm{KNbO}_{3}\right)$ and a perovskite with states in the gap (oxygen deficient $\mathrm{BaNi}_{1 / 2} \mathrm{Nb}_{1 / 2} \mathrm{O}_{3-\delta}$ ), an optimal bandgap for solar light absorption of $1.46 \mathrm{eV}$ for $x=0.1$ was achieved [16]. It followed that under 4 $\mathrm{mW} / \mathrm{cm}^{2}$ illumination by an halogen lamp, $0.7 \mathrm{mV}$ open-circuit photovoltage and $0.1 \mu \mathrm{A} / \mathrm{cm}^{2}$ short-circuit photovoltaic current were measured at room temperature. As impressive, first-principles calculations showed that doping with electronegative ions such as $\mathrm{Bi}^{5+}$ on the $\mathrm{B}$-site of a ferroelectric perovskite such as $\mathrm{KNbO}_{3}(\mathrm{KNO})$ caused intermediate conduction bands to appear in the bandgap, effectively lowering $E_{g}$ from $2.4 \mathrm{eV}$ (calculated bandgap of KNO) to 0.9 $\mathrm{eV}$ in Bi-doped KNO. Such strategy allowed for the design and prediction of large Glass coefficients (20 times larger than those observed and computed in BFO) in solid solutions $\left(\mathrm{Pb}_{2} \mathrm{InNbO}_{6}\right)_{1-x}-\left(\mathrm{Ba}_{2} \mathrm{InNiO}_{6}\right)_{x}$ and $\left(\mathrm{Pb}_{2} \mathrm{ScNbO}_{6}\right)_{1-x}\left(\mathrm{Ba}_{2} \mathrm{ScNiO}_{6}\right)_{x}$ with proper absorption in the visible range [78].

\begin{tabular}{|c|c|}
\hline & Band gap $(\mathrm{eV})$ \\
\hline $\mathrm{BaTiO}_{3}$ & 3.3 \\
$\mathrm{PLZT}(3 / 52 / 48)$ & 3.4 \\
$\mathrm{BiFeO}_{3}$ & $2.2-2.7$ \\
$\mathrm{BiMnO}_{3}[79]$ & 1.1 \\
$\mathrm{Bi}_{2} \mathrm{FeCrO}_{6}[13]$ & $1.4-2.0$ \\
$\mathrm{LiNbO}_{3}[80]$ & 3.78 \\
$\mathrm{KNbO}_{3}[81]$ & 3.3 \\
$(\mathrm{KNO})_{x}\left(\mathrm{BNNO}_{(1-x)}[16]\right.$ & $1.5-3$ \\
$\mathrm{KBiFe}_{2} \mathrm{O}_{5}[64]$ & 1.6 \\
\hline
\end{tabular}

Table 1: Summary of band gaps in various materials exhibiting photovoltaic effects. 


\begin{tabular}{|c|c|c|c|}
\cline { 2 - 4 } \multicolumn{1}{c|}{} & $J_{s c} / \phi_{0}(\mu \mathrm{A} / \mathrm{mW})$ & $V_{o c} / d(\mathrm{kV} / \mathrm{cm})$ & Illumination spectra \\
\hline BaTiO $_{3}[46]$ & $1.5 \times 10^{-5}$ & 0.07 & $488 \mathrm{~nm}$ \\
ITO/PLZT $_{(3 / 52 / 48) / P t}[64]$ & $2.9 \times 10^{-6}$ & 0.14 & $250-1100 \mathrm{~nm}$ \\
ITO/PLZT $_{(3 / 52 / 48)} / \mathrm{Mg}[64]$ & $4.6 \times 10^{-4}$ & 0.27 & $250-1100 \mathrm{~nm}$ \\
WO $_{3}$ doped PLZT $_{(3 / 52 / 48)}$ & $8.8 \times 10^{-5}$ & 2.2 & $366 \mathrm{~nm}$ \\
\hline IT1] & 0.6 & $6.5 \times 10^{-5}$ & $373 \mathrm{~nm}$ \\
\hline (KNO) & & 1.8 & White light \\
\hline
\end{tabular}

Table 2: Summary of different numerical values encountered in the literature for bulk materials. We chose to consider the quantity $\mathrm{J}_{\mathrm{sc}} / \phi_{0}$ with $\phi_{0}$ the illumination surface power density, as many papers show a linear dependency of Jsc on $\phi_{0}$. We also considered th the electric open-circuit field, i.e. the open-circuit voltage Voc divided by the distance between the electrodes $\mathrm{d}$.

\begin{tabular}{|c|c|c|c|}
\hline & $\begin{array}{c}J_{s c} / \phi_{0} \\
(\mu \mathrm{A} / \mathrm{mW})\end{array}$ & $\begin{array}{c}V_{o c} / d \\
(\mathrm{kV} / \mathrm{cm})\end{array}$ & Illumination spectra \\
\hline $\mathrm{BaTiO}_{3-\delta} / \mathrm{Si}$ HOP [68] & NM & 2.65 & $308 \mathrm{~nm}$ \\
\hline $\mathrm{BaTiO}_{3-\delta} / \mathrm{Si}$ MOP [68] & NM & 16.5 & $308 \mathrm{~nm}$ \\
\hline $\mathrm{BaTiO}_{3-\delta} / \mathrm{Si}$ LOP [68] & NM & 18 & $308 \mathrm{~nm}$ \\
\hline $\mathrm{Pt} / \mathrm{BaTiO}_{3}(20-50 \mathrm{~nm}) / \mathrm{Pt}[11]$ & $6 \times 10^{-3}$ & 300 & $360 \mathrm{~nm}$ \\
\hline $\begin{array}{c}\operatorname{ITO}_{\text {PLZT(3/52/48)/Pt/ }} \mathrm{Ti} / \mathrm{SiO}_{2} / \mathrm{Si} \\
{[83]}\end{array}$ & $1.1 \times 10^{-2}$ & 2 & NM \\
\hline ITO/BFO/SRO/STO [62] & 5.3 & $16-18$ & White light \\
\hline $\mathrm{Pt} / \mathrm{Fe} / \mathrm{BFO} / \mathrm{LSMO} / \mathrm{STO}$ [55] & $3 \times 10^{-2}$ & 21 & Halogen lamp \\
\hline BFO (71 DWs) [29] & 0.42 & 0.8 & White light \\
\hline $\mathrm{BFO}[51]$ & 0.08 & 8 & $\begin{array}{l}\text { Solar simulator } \\
\text { (AM 1.5) }\end{array}$ \\
\hline 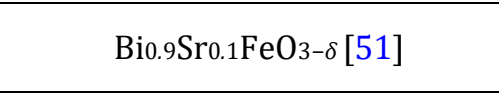 & 0.5 & 14 & $\begin{array}{l}\text { Solar simulator } \\
\text { (AM 1.5) }\end{array}$ \\
\hline BFCO multi-layers [8] & 117 & 89 & $\begin{array}{c}\text { Solar simulator } \\
\text { (AM 1.5) }\end{array}$ \\
\hline $\mathrm{Bi}_{2} \mathrm{FeCrO}_{6}[13]$ & 660 & 59 & $635 \mathrm{~nm}$ \\
\hline
\end{tabular}


Table 3: Summary of different numerical values encountered in the literature for thin films. We chose to consider the quantity $\mathrm{J}_{s c} / \phi_{0}$ with $\phi_{0}$ the illumination surface power density, as many papers show a linear dependency of $\mathrm{J}_{\mathrm{sc}}$ on $\phi$. We also considered the electric open-circuit field, i.e. the open-circuit voltage Voc divided by the distance between the electrodes d. HOP, MOP and LOP stand for High/Middle/Low Oxygen Pressure in the formation process of $\mathrm{BaTiO}_{3} \delta$.

\section{Beyond photovoltaics}

\subsection{Photostriction}

In ferroelectrics, both electric polarization or mechanical strain coexist and are most of the time coupled through electrostriction (quadratic term) and piezoelectricity (linear term). The electric response of ferroelectric materials to light then hold promises towards achieving light-controllable actuators. A recent review has focused on photostrictive materials in general [84] and we shall restrict the present discussion to ferroic aspects of photostriction. This photostriction mechanism (see Figure 13) occurs at two very different time scales, either the second or the picosecond.

\subsubsection{Steady photostriction}

Already back in 1991, Xiao et al. [86] showed that $\mathrm{Fe}: \mathrm{LiNbO}_{3}$ exhibits strain of $0.04 \%$ after $10 \mathrm{~min}$ of white light illumination with a $100 \mathrm{~mW} / \mathrm{cm}^{2}$ incident power. Later, BFO was shown to exhibit a similar behavior, but with time responses (0.1-1 s) and amplitude (0.001\%) one order of magnitude smaller [87]. Possible thermal expansion effects have up to now been ruled out due to incompatible time scales [87]. Using a capacitance dilatometer, it was showed that upon shining light on the (101) (pseudo-cubic notation) face of a single cystal BFO, it elongates along [010] [85], and conversely, shining light on the (010) face stretches BFO along [101] [87]. In the latter case, BFO showed enhanced photoresponse for the wavelengths of $530 \mathrm{~nm}$ (2.4 eV energy, near the band gap edge) and $365 \mathrm{~nm}$, and white light showed also an enhanced photoresponse compared to monochromatic visible light. Experiments with linearly polarized 
light showed a sinusoidal variation of the photo-induced strain, directly hinting at the BPVE $[10,23]$ coupled with the inverse piezoelectric effect as the main mechanisms behind photostrictive effects. Interestingly, because BFO is magnetoelectric (polarization and magnetization are coupled), the photostrain can be also modulated using an external magnetic field [85].
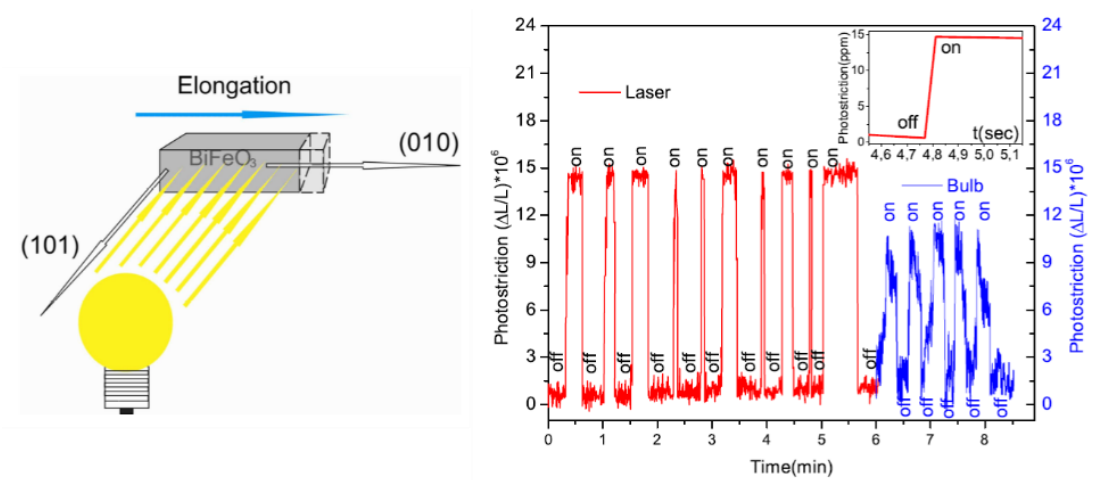

Figure 13: On the left, sketch of the experimental procedure [85]; On the right, relative length variation of the BFO single domain crystal in the (010) direction when irradiating with a HeNe laser (red) or with a white light bulb (blue). Taken from [85].

\subsubsection{Transient ultra-fast photoinduced mechanical response}

In pump-probe technique experiments, a pump laser is shone on short time scales (ps or fs), and a probe laser probes the state of the material. X-ray diffraction have been used as a probe while a 50 fs laser pulse with central peak wavelength at $400 \mathrm{~nm}$ as the pump, and the shift of the (002) peak of $35 \mathrm{~nm}$ thick (001)-BFO thin films is monitored [88]. A shift of the (002) Bragg peaks is detected within $100 \mathrm{ps}$, the time resolution of the experiment, and then decays bi-exponentially towards its initial state with both a fast $(\approx 2 \mathrm{~ns})$ and a slow $(\approx$ $63 \mathrm{~ns}$ ) time constants. The initial shift of the (002) peak can be as high as $0.08^{\circ}$ at a laser fluence of $3.2 \mathrm{~mJ} / \mathrm{cm}^{2}$ (see Figure 14), resulting in a strain $\Delta c / c=0.41$ \%. Additionally, transient absorption measurements showed that a linear 
relationship holds between the concentration of photo-induced carriers and the induced strain.
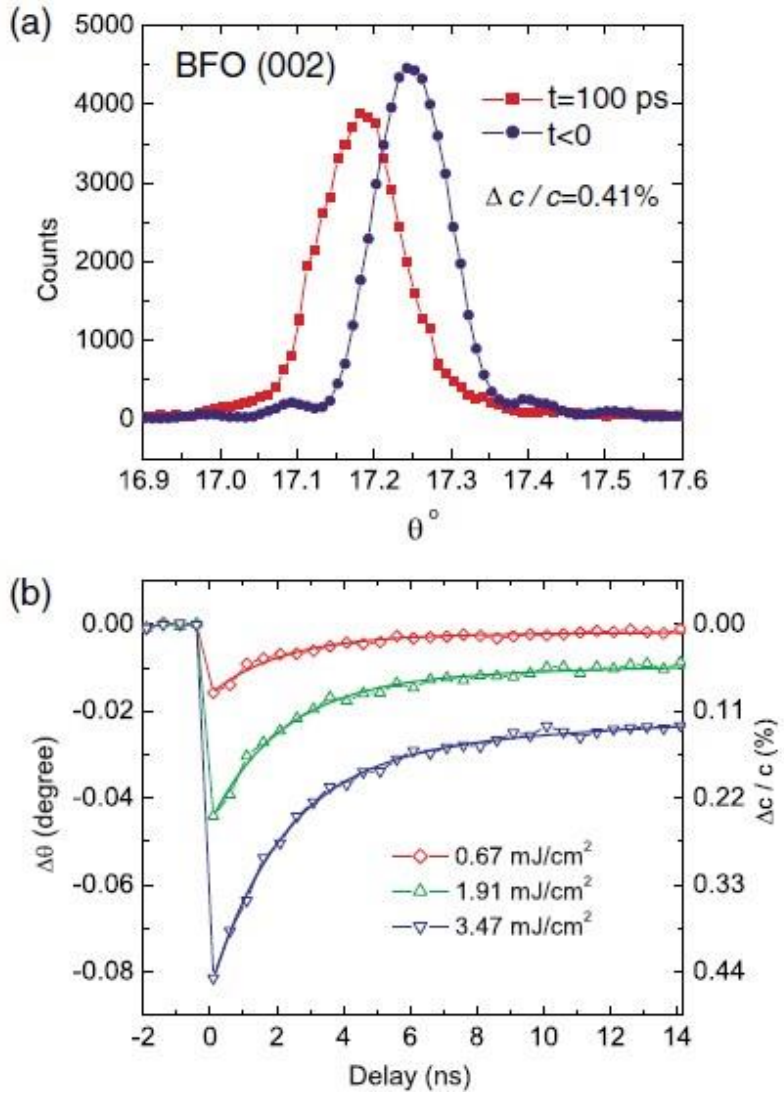

Figure 14: (a) BFO (002) Bragg peak. (b) Shift of the BFO (002) Bragg peak $\Delta \theta$ and corresponding strain variation. Reproduced with permission from [88]. Copyrighted by the American Physical Society.

These results were further confirmed with a time resolution of $100 \mathrm{fs}$, and the broadening of (002) peak was explained by a strain gradient within the thickness of the film [89]. The appearance of photoinduced strain is only limited by the sound velocity of BFO, estimated to $3500 \mathrm{~m} / \mathrm{s}$. In lead titanate under illumination, an instantaneous increase in tetragonality that decays over a characteristic time of the order of nanoseconds was reported [90]. 
The pump-probe technique can also be used to study acoustic phonons [91], which excited gigahertz acoustic modes using a UV pump of $3.1 \mathrm{eV}$ and a probe of $1.55 \mathrm{eV}$. Interestingly, it has been shown that BFO exhibits a strong shear acoustic mode as compared to ordinary semi-conductors [92].

In most of those studies, the photostrictive effect is explained as a combination of photovoltaic and inverse piezoelectric effects, as it accounts for the large shear stress observed in BFO [92]. Electrostriction and thermal expansion have most of the time been ruled out. As free carriers are generated by light, they will eventually screen the polarization, inducing a change in the depolarizing field. This in turn induces a strain via the inverse piezoelectric effect. Note however that a very recent study of optically-induced dynamic strain gradients in $\mathrm{BiFeO}_{3}$ thinfilms has led the authors to propose a new mechanism, based on exciton diffusion and dissociation into screening free carriers at the interfaces, for the ultra-fast photoinduced mechanical response [93]. Nonetheless, the few studies of transient photodynamics in ferroelectrics up to day all agree that the screening of internal fields and the subsequent inverse piezoelectric effect are responsible for this ultra-fast light-induced mechanical response.

\subsection{Photocatalytic Activity}

Among semiconductors considered for photocatalysis [94, 95], the most used and investigated is $\mathrm{TiO}_{2}$. However, several issues limit its efficiency: the wide band gap, the high recombination rate of the photogenerated charge carriers, photostability in water, etc. Notably in $\mathrm{TiO}_{2}$, only $4 \%$ of the total solar spectrum can photoexcite charge carriers, conditioning the need for artificial UV radiation to drive efficiently the chemical reactions. Photovoltaic ferroelectrics might be considered as an alternative choice as most of them are non-toxic oxides, stable in water and crystallize in the perovskite structure which is close to that of $\mathrm{TiO}_{2}$. In ferroelectrics, once the light excitation generates electrons-hole pairs, the polarization acts like an internal electric field favoring separation of the photo- 
induced charges carriers and thus increasing their lifetime by reducing their recombination. This is for instance revealed by the much larger decay time of photoluminescence in ferroelectric $\mathrm{LiNbO}_{3}$ as compared to non-ferroelectric $\mathrm{TiO}_{2}$ [96] (9 $\mu$ s against $\left.0.1 \mu \mathrm{s}\right)$.

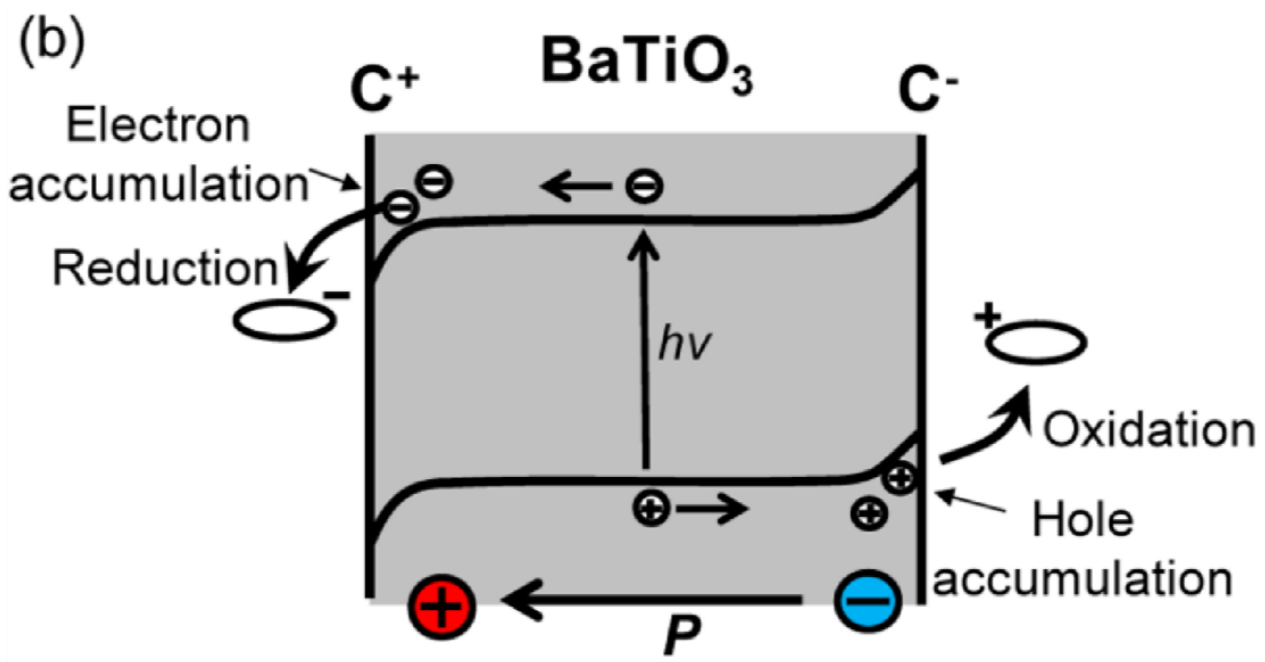

Figure 15: Band bending caused by the polarization in $\mathrm{BaTiO}_{3}$ and the free photoexcited carriers act jointly to enhance redox chemical reactions. Reprinted with permission from Ref. [101]. Copyright 2013 American Chemical Society.

Interestingly, photogenerated charges can migrate to the material surface (see Figure 15) and serve as efficient redox reaction sources for the degradation of contaminant molecules $[97,98]$ in wastewater treatment as well as water splitting $[99,100]$ in sustainable hydrogen fuel cells. When using photodecolorization of a target dye-molecule, the decolorization rate is significantly enhanced when ferroelectric $\mathrm{BaTiO}_{3}$ particles rather than nonferroelectric ones are used [101]. A full (100\% efficiency) photocatalytic degradation of the molecule is achieved after 40 min illumination under solar simulator using ferroelectric $\mathrm{BaTiO}_{3}$ particles coated by Ag nanoparticles [101] (see Figure 16). Similarly photocatalytic effects of $\mathrm{BiFeO}_{3}$ to oxidize organic dyes was demonstrated not only using UV irradiation [102] but also visible light [97, 103] thanks to its lower band gap. Moreover in ferroelectrics, the appearance of 
polarization gives rise to surface charges which in turn induce a depolarizing field that acts against the polarization. Adsorption or chemisorption of molecules onto the surface can minimize the effect of the depolarizing field by screening the polarization charges $[104,105]$. As a result a strong Stern layer can be formed favoring interactions with environment reactants. A stronger adsorption of dye molecules was observed in $\mathrm{LiNbO}_{3}$ in comparison to $\mathrm{TiO}_{2}$ powders $(7.79 \%$ per unit area against 1.27\%) [106]. By switching the polarization direction and thus the type of polarization charges at the surface of the ferroelectric, it is also possible to preferentially favor a type of reactions. For instance, $\mathrm{H}_{2}$ production is enhanced on the positive surface of $\mathrm{Sr}$-doped $\mathrm{Pb}(\mathrm{Zr}, \mathrm{Ti}) \mathrm{O}_{3}$ [107] while negative polarization charges are found to promote the oxidation reaction both experimentally and theoretically in case of $\mathrm{BiFeO}_{3}$ [108]. Interestingly, it was also proposed that molecules having (permanent or induced) dipole moment can interact with the polarization at the surface of the ferroelectric and reduces the energy required to break bonds which therefore enhances the photochemical activity [109]. Finally, we note that a piezo-electrochemical effect has been reported as an efficient photocatalytic mechanism in $\mathrm{BaTiO}_{3}$ dendrites [110] suggesting that other cross-coupled processes could exist. 


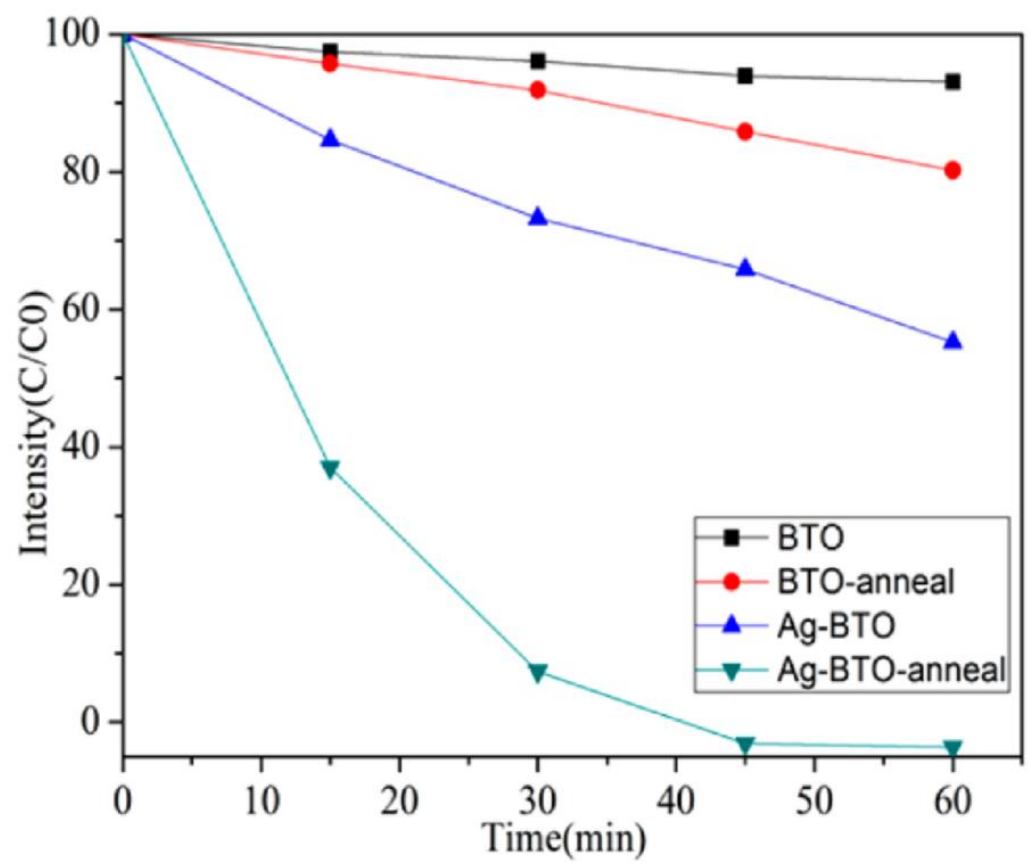

Figure 16: Ag nanoparticles coated $\mathrm{BaTiO}_{3}$ are 7.25 times faster than bare BaTiO3 in degrading the dye molecule Rhodamine B. Reprinted with permission from Ref. [101]. Copyright 2013 American Chemical Society.

\section{Conclusion}

In this paper we review the current status of photovoltaic ferroelectrics and identify critical factors that need to be further explored and challenged, both experimentally and theoretically, in order to achieve a better fundamental understanding as well as higher-efficiency. $\mathrm{ABX}_{3}$ perovskites are the most investigated ferroelectrics under illumination. The perovskite structure is characterized by the ability to incorporate on each of the A and/or B sites a large variety of different cations. This flexibility of the perovskite structure allows for tuning of the band gap by doping or creating solid solutions, in order to achieve better absorption of the solar spectrum (see Tables 1 for a summary of the bandgap of the current photoferroelectrics). The effect of various types of chemical order/disorder in such solid solutions on photo-properties has yet to be 
studied in more details. Different types of charge ordering, their geometries (linear, planar etc.) but also the potential benefits between short-range or longrange order are worth being investigated. The integration of different solid solution in heterostructure has proved to be one of the most viable routes towards high PCE photovoltaics devices [8], by achieving an 8.1\% yield. Other structures than perovskite, like Aurivillius $\left[\left(\mathrm{Bi}_{2} \mathrm{O}_{2}\right)\left(\mathrm{A}_{n-1} \mathrm{~B}_{n} \mathrm{O}_{3 n+1}\right.\right.$, e.g. $\mathrm{Bi}_{4} \mathrm{Ti}_{3} \mathrm{O}_{1} 2$ or $\mathrm{SrBi}_{2} \mathrm{Ta}_{2} \mathrm{O}_{9}$ ] or tungsten-bronze [e.g. $\mathrm{Na}_{x} \mathrm{WO}_{3}$ or $\mathrm{Sr}_{x} \mathrm{Ba}_{1-x} \mathrm{Nb}_{2} \mathrm{O}_{6}$ (SBN) or $\mathrm{Ba}_{2} \mathrm{NaNb}_{5} \mathrm{O}_{1} 5$ (BNN)] phases are worth being explored.

Most ferroelectrics undergo phase transitions towards paraelectric or other ferroelectric states. These phase transitions are accompanied by polar and strain changes, which are expected to drastically affect photo-ferroelectric properties. To the best of our knowledge, this has not yet been systematically explored. Ferroelectrics are strain-sensitive, and as a consequence all types of deformation (uniaxial, biaxial, isotropic etc.) are expected to affect photo-induced properties, allowing to envisage the systematic use of strain-engineering, specifically in thin films (see Table $3 \& 2$ for a comparison of bulk and thin films photovoltaic effects). The effect of size and consequently the way of organizing the ferroelectric domains should be addressed in close link with the diffusion length of the photo-generated charges carriers that still has to be determined. The anisotropy and thus the shape of the ferroelectric when subjected to light irradiation is also an important aspect to be considered as the photo-induced effects have tensorial properties. Efforts in determining generation and recombination rates and their mechanisms in photovoltaic ferroelectrics should be realized and mechanisms at different time scales should be more investigated as for instance the steady photo-deformation is $0.001 \%$ while it reaches $0.41 \%$ in ultra-fast measurements. The role of the interface should be widely addressed through various transparent electrodes, electron and/or hole transporter media, multilayers and superlattices structures or core-shell structures to enhance photo-voltaic/-strictive/-catalytic activity. A peculiar interest on the band structure and bending to reveal the whole features of the electronic structure 
should be then considered for designing more efficient photo-electronic devices. Defects (space charge, polarons, ordered defects,...) which are also known to contribute in classical photovoltaics require further investigation efforts to fully understand their roles and their consequences simultaneously on both the polarization and the light interactions. More links between processes associated with photovoltaics like photostriction, photocatalysis, photolysis and others should provide much better understanding and innovative concepts. Similarly, cross-coupled mechanisms such as the piezo-electrochemical are interesting to be investigated. One may wonder how polarization, strain, magnetization and chemistry interact all together with light in multiferroics. This should also lead to novel devices triggered or assisted by light excitation. Finally while the field of photovoltaic ferroelectrics is still in its infancy, the accumulated knowledge and its links with other fields has the potential to inspire other materials communities. Among them, the recent organo-metal-halide perovskite solar cells which have known an unprecedented increase in PCE from 3.8\% to over 20\% [111] since 2009, and may largely benefit from the historic knowledge in ferroelectrics. Indeed while ferroelectric contribution has been excluded in the switchable photovoltaic effect [112] in $\mathrm{MAPbI}_{3}$, first achievements include the recent demonstration of switchable ferroelectric domains using piezoresponse force microscopy [113] and the theoretical prediction from first principles that charged domain walls may be used to reduce the band gap (see Figure 6 of Ref. [114]). Generally speaking, the convergence of two fields of research, can considerably accelerate the development of each of the domains, as recently amply demonstrated in the field of multiferroics, bridging the fields of ferroelectricity and magnetism. We are convinced that a similar convergence between ferroelectrics and the field of semiconductor photovoltaics will equally lead to new, exciting and useful discoveries. 


\section{$5 \quad$ Acknowledgements}

C.P. thanks a public grant overseen by the French National Research Agency (ANR) as part of the "Investissements d'Avenir" program (reference: ANR-10LABX-0035, Labex NanoSaclay). J.K. and M.G. acknowledge financial support from the Fonds National de Recherche Luxembourgeois through a PEARL grant FNR/P12/48J315J/Kreisel.

\section{References}

[1] M. E. Lines, A. M. Glass, Principles and Applications of Ferroelectrics and Related Materials, Oxford University Press, New York, 1977

[2] L. Su, L. Zou, C.-C. Fong, W.-L. Wong, F. Wei, K.-Y. Wong, R. S. S. Wu, M. Yang, Biosens. Bioelec. 2013, 46, 155

[3] K. Uchino, Ferroelectric Devices, CRC Press, Boca Raton, 2010

[4] S.-W. Cheong, M. Mostovoy Nat. Mater. 2007, 6, 13

[5] R. Rameshn N. A. Spaldin Nat. Mater. 2007, 6, 21

[6] T. Choi, S. Lee, Y. J. Choi, V. Kiryukin, S.-W. Cheong, Science 2009, 324, 63

[7] V. M. Fridkin, R. M. Magomadov, Pis'ma Zh. Eksp. Teor. Fiz. 1979, 30, 723

[8] R. Nechache, C. Harnagea, S. Li, L. Cardenas, W. Huang, J. Chakrabartty, F. Rosei, Nat. Photon. 2014, 9, 61

[9] W. Shockley, H. J. Queisser, J. Appl. Phys. 1961, 32, 510

[10] V. M. Fridkin, Cryst. Rep. 2001, 46, 654

[11] A. Zenkevich, Y. Matveyev, K. Maksimova, R. Gaynutdinov, A. Tolstikhina, V. M. Fridkin, Phys. Rev. B 2014, 90, 161409 
[12] M. Qin, K. Yao, Y. C. Liang, Appl. Phys. Lett. 2009, 95, 022912

[13] R. Nechache, C. Harnagea, S. Licoccia, E. Traversa, A. Ruediger, A. Pignolet, F. Rosei, Appl. Phys. Lett. 2011, 98, 202902

[14] M. Ichiki, H. Furue, T. Kobayashi, R. Maeda, Y. Morikawa, T. Nakada, K. Nonaka, Appl. Phys. Lett. 2005, 87, 222903

[15] M. Qin, K. Yao, Y. C. Liang, Appl. Phys. Lett. 2008, 93, 122904

[16] I. Grinberg, V. West, M. Torres, G. Gou, D. M. Stein, L. Wu, G. Chen, E. M. Gallo, A. R. Akbashev, P. K. Davies, J. E. Spanier, A. M. Rappe, Nature 2013, 503, 509

[17] A. G. Chynoweth, Phys. Rev. 1956, 102, 705.

[18] V. M. Fridkin, A. A. Grekov, N. A. Kosonogov, Ferroelectrics 1972, 4, 915.

[19] A. M. Glass, D. von der Linde, T. J. Negran, Appl. Phys. Lett. 1974, 25, 233

[20] A. M. Glass, D. von der Linde, T. J. Negran, J. Elec. Mat. 1975, 4, 915

[21] G. Chanussot, A. M. Glass, Phys. Lett. A 1976, 59, 405

[22] R. von Baltz, W. Kraut, Sol. State Comm. 1978, 26, 961

[23] B. I. Sturman, V. M. Fridkin, The Photovoltaic and Photorefractive Effects in Noncentrosymmetric Materials, Gordon and Breach Science Publishers,

\subsection{2}

[24] W. Kraut, R. von Baltz, Phys. Rev. B 1979, 19

[25] R. von Baltz, W. Kraut, Phys. Rev. B 1981, 10

[26] V. M. Fridkin, E. P. Efremova, B. H. Karimov, Appl. Phys. 1981, 80, 77 
[27] S. M. Young, A. M. Rappe, Phys. Rev. Lett. 2012, 109, 116601

[28] S. M. Young, F. Zheng, A. M. Rappe, Phys. Rev. Lett. 2013, 110, 057201

[29] S. Y. Yang, Jan Seidel, S. J. Byrnes, P Shafer, C.-H. Yang, M. D. Rossell, P. Yu, Y.-H. Chu, J. F. Scott, J. W. Ager, L. W. Martin, R. Ramesh, Nat. Nanotech. 2010, 5, 143

[30] R. Moubah, O. Rousseau, D. Colson, A. Artemenko, M. Maglione, M. Viret, Adv. Func. Mater. 2012, 22, 4814

[31] J. Kreisel, M. Alexe, P. A. Thomas, Nat. Mat. 2012, 11, 260

[32] G. Catalan, J. Seidel, R. Ramesh, J. F. Scott, Rev. Mod. Phys. 2012, 84, 119

[33] T. Sluka, A. K. Tagantsev, P. Bednyakov, N. Setter, Nat. Comm. 2013, 4,1808

[34] J. Guyonnet, I. Gaponenko, S. Garigliano, P. Paruch Adv. Mater. 2011, 23,5377

[35] A. Lubk, S. Gemming, N. Spaldin, Phys. Rev. B 2009, 80, 104110

[36] J. Seidel, S. Y. Yang, E. Alarc'on-Llad'o, J. Wu, R. Ramesh, J. W. Ager, Ferroelectrics 2012, 433, 123

[37] J. Seidel, D. Fu, S. Y. Yang, E. Alarc'on-Llad'o, J. Wu, R. Ramesh, J. W. Ager, Phys. Rev. Lett. 2011, 107, 126805

[38] O. Di'eguez, P. Aguado-Puente, J. Junquera, J. Iniguez, Phys. Rev. B 2013, 87, 024102

[39] M. Alexe, D. Hesse, Nat. Comm. 2011, 2, 256

[40] M. Alexe, Nano Letters 2012, 12, 2193

[41] W. Ji, K. Yao, Y. Liang,Phys. Rev. B 2011, 84, 094115 
[42] A. Bhatnagar, A. R. Chaudhuri, Y. H. Kim, D. Hesse, M. Alexe, Nat. Comm. 2013, 4, 2835

[43] S. Farokhipoor, B. Noheda Phys. Rev. Lett. 2011, 107, 127601

[44] J. Seidel, L. W. Martin, Q. He, Q. Zhan, Y.-H. Chu, A. Rother, M. E. Hawkridge, P. Maksymovych, P. Yu, M. Gajek, N. Balke, S. V. Kalinin, S. Gemming, F. Wang, G. Catalan, J. F. Scott, N. A. Spaldin, J. Orenstein, R. Ramesh, Nat. Mat. 2009, 8, 229

[45] J. Seidel, G. Singh-Bhalla, Q. He, S.-Y. Yang, Y.-H. Chu, R. Ramesh, Phase Trans. 2013, 86, 53

[46] W. T. H. Koch, R. Munser, W. Ruppel, P. Würfel, Sol. State Comm. 1975, 17, 847

[47] S. M. Young, F. Zheng, A. M. Rappe, Phys. Rev. Lett. 2012, 109, 236601

[48] W. Wu, J. R. Guest, Y. Horibe, S. Park, T. Choi, S.-W. Cheong, M. Bode, Phys. Rev. Lett. 2010, 104, 217601

[49] C. Ge, K.-J. Jin, C. Wang, H.-B. Lu, C. Wang, G. Yang, Appl. Phys. Lett. 2011, 99, 063509

[50] C. Ge, K.-J. Jin, C. Wang, H.-B. Lu, G. Yang, Nano-Micro Lett. 2013, 5, 81

[51] Y. Guo, B. Guo, W. Dong, H. Li, H. Liu, Nanotech. 2013, 24, 275201

[52] D. Lee, S. H. Baek, T. H. Kim, J.-G. Yoon, C. M. Folkman, C. B. Eom, T. W. Noh, Phys. Rev. B 2011, 84, 125305

[53] R. Guo, L. You, L. Chen, D. Wu, J. Wang, Appl. Phys. Lett. 2011, 99, 129902

[54] K. Yao, B. K. Gan, M. Chen, S. Shannigrahi, Appl. Phys. Lett. 2005, 87, 212906 
[55] R. Guo, L. You, Y. Zhou, A. S. Lim, X. Zou, L. Chen, R. Ramesh, J. Wang, Nat. Comm. 2013, 4, 1990

[56] G. Chanussot, V. M. Fridkin, G. Godefroy, B. Jannot, Appl. Phys. Lett. $1977,31,3$

[57] K. Toda, M. Oikawa, K. Hisano, Appl. Phys. 1978, 209, 207

[58] P. W. M. Blom, R. M. Wolf, J. F. M. Cillessen, M. Krijn, Phys. Rev. Lett. 1994, 73, 2107

[59] L. Pintilie, I. Boerasu, M. J. M. Gomes, T. Zhao, R. Ramesh, M. Alexe, J. Appl. Phys. 2005, 98, 124104

[60] L. Pintilie, M. Alexe, J. Appl. Phys. 2005, 98, 124103

[61] G.-L. Yuan, J. Wang, Appl. Phys. Lett. 2009, 95, 252904

[62] S. Y. Yang, L. W. Martin, S. J. Byrnes, T. E. Conry, S. R. Basu, D. Paran, L. Reichertz, J. Ihlefeld, C. Adamo, A. Melville, Y.-H. Chu, C.-H. Yang, J. L. Musfeldt, D. G. Schlom, J. W. Ager, R. Ramesh, Appl. Phys. Lett. 2009, 95, 062909

[63] L. Pintilie, C. Dragoi, I. Pintilie, J. Appl. Phys. 2011, 110, 044105

[64] J. Zhang, X. Su, M. Shen, Z. Dai, L. Zhang, X. He, W. Cheng, M. Cao, G. Zou, Sci. Rep. 2013, 3, 2109

[65] V. N. Harshan, S. Kotru, Appl. Phys. Lett. 2012, 100, 173901

[66] F. Liang, L. You, Y. Zhou, P. Ren, Z. S. Lim, J. Wang, Appl. Phys. Lett. 2014, 104, 142903

[67] H. T. Yi, T. Choi, S. G. Choi, Y. S. Oh, S.-W. Cheong, Adv. Mat. 2011, 23, 3403

[68] J. Xing, K.-J. Jin, H. Lu, M. He, G. Liu, J. Qiu, G. Yang, Appl. Phys. Lett. 2008, 92, 071113 
[69] V. K. Yarmarkin, B. M. Gol'tsman, M. M. Kazanin, V. V. Lemanov, Phys. Sol. State 2000, 42, 522

[70] M. Yang, A. Bhatnagar, M. Alexe, Adv. El. Mater. 2015, in press

[71] P. Poosanaas, A. Dogan, S. Thakoor, K. Uchino, J. Appl. Phys., 84, 1508

[72] P. S. Brody, F. Crowne, J. Elec. Mater. 1975, 4, 955

[73] P. S. Brody, J. Sol. State Chem. 1975, 12, 193

[74] J. Chakrabartty, R. Nechache, S. Li, M. Nicklaus, A. Ruediger, F. Rosei, J. Am. Ceram. Soc. 2014, 97, 1837

[75] T. Qi, I. Grinberg, A. M. Rappe, Phys. Rev. B 2011, 83, 224108

[76] F. Wang, I. Grinberg, A. M. Rappe, Appl. Phys. Lett. 2014, 104, 152903 [77] A. De Vos, J. Phys. D: Appl. Phys. 1980, 13, 839

[78] L. Jiang, I. Grinberg, F. Wang, S. M. Young, P. K. Davies, A. M. Rappe Phys. Rev. B 2014, 90, 075153

[79] J. P. Chakrabartty, R. Nechache, C. Harnagea, F. Rosei, Opt. Expr. 2013, 22, A80

[80] A. Dhar, A. Mansingh, J. Appl. Phys. 1990, 68, 5804

[81] E. Wiesandanger, Ferroelectrics 1973, 6, 263

[82] C.-M. Hung, C.-S. Tu, W. D. Yen, L. S. Jou, M.-D. Jiang, V. H. Schmidt, J. Appl. Phys. 2012, 111, 07D912

[83] M. Ichiki, R. Maeda, Y. Morikawa, Y. Mabune, T. Nakada, K. Nonaka, Appl. Phys. Lett. 2004, 84, 395

[84] B. Kundys, Appl. Phys. Rev. 2015, 1, 011301

[85] B. Kundys, M. Viret, D. Colson, D. O. Kundys, Nat. Mater. 2010, 9, 803 
[86] X. Dingquan, Z. Jianguo, Z. Shipin, W. Xiu, Sol. State Comm. 1991, 79, 1005

[87] B. Kundys, M. Viret, C. Meny, V. Da Costa, D. Colson, B. Doulin, Phys. Rev. B 2012, 85, 092301

[88] H. Wen, P. Chen, M. P. Cosgriff, D. A. Walko, J. H. Lee, C. Adamo, R. D. Schaller, J. F. Ihlefeld, E. M. Dufresne, D. G. Schlom, P. G. Evans, J. W. Freeland, Y. Li, Phys. Rev. Lett. 2013, 110, 037601

[89] D. Schick, M. Herzog, H. Wen, P. Chen, C. Adamo, P. Gaal, D. G. Schlom, P. G. Evans, Y. Li, M. Bargheer, Phys. Rev. Lett. 2014, 112, 097602

[90] D. Daranciang, M. J. Highland, H. Wen, S. M. Young, N. C. Brandt, H. Y. Hwang, M. Vattilana, F. Quirin, J. Goodfellow, T. Qi, I. Grinberg, D. M. Fritz, M. Cammarata, D. Zhu, H. T. Lemke, D. Walko, E. M. Dufresne, Y. Li, J. Larsson, D. A. Reis, K. Sokolowski-Tinten, K. A. Nelson, A. M. Rappe, P. H. Fuoss, G. B. Stephenson, A. M. Lindenberg, Phys. Rev. Lett. 2012, 108, 087601

[91] P. Ruello, T. Pezeril, S. Avanesyan, G. Vaudel, V. E. Gusev, I. C. Infante, B. Dkhil, Appl. Phys. Lett. 2012, 100, 212906

[92] M. Lejman, G. Vaudel, I. C. Infante, P. Gemeiner, V. E. Gusev, B. Dkhil, P. Ruello, Nat. Comm. 2014, 5, 4301

[93] Y. Li, C. Adamo, P. Chen, P. G. Evans, D. G. Schlom, D. A. Walko, H. Wen, Q. Zhang, Sci. Rep. 2015, accepted.

[94] C. E. Nebel, Nat. Mater. 2013, 12, 780

[95] Y. Qu, X. Duan, Chem. Soc. Rev. 2013, 42, 2568

[96] N. J. Bell, Y. H. Ng, A. Du, H. Coster, S. C. Smith, R. Amal, J. Phys. Chem. C 2011, 115,6004

[97] F. Gao, X. Y. Chen, K. B. Yin, S. Dong, Z. F. Ren, F. Yuan, T. Yu, Z. 
G. Zou, J.-M. Liu, Adv. Mater. 2007, 19, 2889

[98] S. Zhang, P. Audebert, Y. Wei, J.-S. Lauret, L. Galmiche, E. Deleporte, J. Mater. Chem. 2011, 21, 466

[99] J. Deng, S. Banerjee, S. K. Mohapatra, Y. R. Smith, M. Misra, J. Fund. Renewable Energy and Applications 2011, 1, R101204

[100] X. W. Lou, Y. Wang, C. Yuan, J. Y. Lee, L. A. Archer, Adv. Mater. 2006 18,2325

[101] Y. Cui, J. Briscoe, S. Dunn, Chem. Mater. 2013, 25, 4215

[102] Z. Liu, Y. Qi, C. Lu, J. Mater. Sci.: Mater. Electron. 2010, 21, 380

[103] S. Li, Y.-H. Lin, B.-P. Zhang, C.-W. Nan, Y. Wang, J. Appl. Phys. 2009, 105, 056105

[104] D. Li, M. H. Zhao, J. Garra, A. M. Kolpak, A. M. Rappe, D. A. Bonnell, J. M. Vohs, Nat. Mater. 2008, 7, 473

[105] J. Shin, V. B. Nascimento, G. Geneste, J. Rundgren, E. Ward Plummer, B. Dkhil, S. V. Kalinin, A. P. Baddorf, Nano Letters 2009, 9, 3720

[106] M. Stock, S. Dunn, J. Phys. Chem. C 2012, 116, 20854

[107] Y. Inoue, Energy Environ. Sci. 2009, 2, 364

[108] W. Ji, K. Yao, Y.-F. Lim, Y. C. Liang, A. Suwardi, Appl. Phys. Lett. 2013, 103, 062901

[109] M. Stock, IEEE Ultrasonic, Ferroelectrics, and Frequency Control 2011, 58, 1988

[110] K.-S. Hong, H. Xu, H. Konishi, X. Li, J. Phys. Chem. C 2012, 116, 13045 [111] N.-G. Park, Materials Today 2015, 18, 65 
[112] Z. Xiao, Y. Yuan, Y. Shao, Q. Wang, Q. Dong, C. Bi, P. Sharma, A. Gruverman, J. Huang, Nat. Mater. 2014, 14, 193

[113] Y. Kutes, L. Ye, Y. Zhou, S. Pang, B. D. Huey, N. P. Padture, J. Phys.

Chem. Lett. 2014, 5, 3335

[114] S. Liu, F. Zheng, N. Z. Koocher, H. Takenaka, F. Wang, A. M. Rappe, J. Phys. Chem. Lett. C 2015, 6, 693 